\title{
An exact local conservation theorem for finite-amplitude disturbances to non- parallel shear flows, with remarks on Hamiltonian structure and on Arnol'd's stability theorems
}

Article

Published Version

Mcintyre, M. E. and Shepherd, T. G. (1987) An exact local conservation theorem for finite-amplitude disturbances to nonparallel shear flows, with remarks on Hamiltonian structure and on Arnol'd's stability theorems. Journal of Fluid Mechanics, 181 (1). p. 527. ISSN 0022-1120 doi: https://doi.org/10.1017/S0022112087002209 Available at https://centaur.reading.ac.uk/32993/

It is advisable to refer to the publisher's version if you intend to cite from the work. See Guidance on citing.

Published version at: http://dx.doi.org/10.1017/S0022112087002209

To link to this article DOI: http://dx.doi.org/10.1017/S0022112087002209

Publisher: Cambridge University Press

All outputs in CentAUR are protected by Intellectual Property Rights law, including copyright law. Copyright and IPR is retained by the creators or other copyright holders. Terms and conditions for use of this material are defined in the End User Agreement. 


\section{www.reading.ac.uk/centaur}

\section{CentAUR}

Central Archive at the University of Reading

Reading's research outputs online 


\title{
An exact local conservation theorem for finite-amplitude disturbances to non-parallel shear flows, with remarks on Hamiltonian structure and on Arnol'd's stability theorems
}

\author{
By M. E. MCINTYRE AND T. G. SHEPHERD \\ Department of Applied Mathematics and Theoretical Physics, University of Cambridge, \\ Silver Street, Cambridge CB3 9EW, UK
}

(Received 20 September 1985 and in revised form 15 August 1986)

Disturbances of arbitrary amplitude are superposed on a basic flow which is assumed to be steady and either (a) two-dimensional, homogeneous, and incompressible (rotating or non-rotating) or (b) stably stratified and quasi-geostrophic. Flow over shallow topography is allowed in either case. The basic flow, as well as the disturbance, is assumed to be subject neither to external forcing nor to dissipative processes like viscosity. An exact, local 'wave-activity conservation theorem' is derived in which the density $\boldsymbol{A}$ and flux $\boldsymbol{F}$ are second-order 'wave properties' or 'disturbance properties', meaning that they are $O\left(a^{2}\right)$ in magnitude as disturbance amplitude $a \rightarrow 0$, and that they are evaluable correct to $O\left(a^{2}\right)$ from linear theory, to $O\left(a^{3}\right)$ from second-order theory, and so on to higher orders in $a$. For a disturbance in the form of a single, slowly varying, non-stationary Rossby wavetrain, $\bar{F} / \bar{A}$ reduces approximately to the Rossby-wave group velocity, where ( ) is an appropriate averaging operator. $F$ and $A$ have the formal appearance of Eulerian quantities, but generally involve a multivalued function the correct branch of which requires a certain amount of Lagrangian information for its determination. It is shown that, in a certain sense, the construction of conservable, quasi-Eulerian wave properties like $A$ is unique and that the multivaluedness is inescapable in general. The connection with the concepts of pseudoenergy (quasi-energy), pseudomomentum (quasi-momentum), and 'Eliassen-Palm wave activity' is noted.

The relationship of this and similar conservation theorems to dynamical fundamentals and to Arnol'd's nonlinear stability theorems is discussed in the light of recent advances in Hamiltonian dynamics. These show where such conservation theorems come from and how to construct them in other cases. An elementary proof of the Hamiltonian structure of two-dimensional Eulerian vortex dynamics is put on record, with explicit attention to the boundary conditions. The connection between Arnol'd's second stability theorem and the suppression of shear and self-tuning resonant instabilities by boundary constraints is discussed, and a finite-amplitude counterpart to Rayleigh's inflection-point theorem noted.

\section{Introduction}

Andrews (1983) has recently presented a local conservation theorem for small disturbances to a certain class of steady, non-parallel shear flows. The theorem is 
expressed purely in terms of Eulerian quantities, and has potential application inter alia to the study of Rossby waves (vorticity waves) in the atmosphere and oceans. For instance, the flux whose divergence appears in the conservation theorem may be used to quantify the amount of Rossby-wave activity propagating from one place to another, provided that the waves have non-vanishing phase speed relative to the steady basic flow; for further discussion see Plumb $(1985 a, b)$. Propagation in a highly inhomogeneous, moving medium is involved, so that standard concepts like energy flux, or group velocity and wave-action flux for slowly varying wavetrains, may fail to apply.

However, Andrews' result is restricted to disturbances of infinitesimal amplitude, and this may be a severe limitation in practice. For instance, atmospheric Rossby waves typically attain large amplitudes; indeed they may 'break' in a certain sense which seems fundamentally significant (e.g. McIntyre \& Palmer 1983, 1984, 1985) and which includes the breaking of waves on the boundaries of vortex patches previously remarked on by Deem \& Zabusky (1978). Examples are shown in figure 4 below. Recognition of the dynamical linkages associated with Rossby-wave propagation and breaking appears to be important for an improved understanding of the large-scale atmospheric circulation. As a further step towards developing the theoretical tools needed to quantify these ideas, this paper points out the exact, finite-amplitude generalization of Andrews' result ( $\$ 2-4)$, discusses the extent to which it may be regarded as an Eulerian result $(\$ 5)$ and sets it in the context of related theoretical work $(\$ \$ 6,7)$.

The results involve the functional relation between the basic-state stream function $\Psi$ and vorticity $Q$ which arises from the condition $\partial(\Psi, Q)=0$ for an inviscid, unforced, two-dimensional, incompressible, steady basic flow. Here $\partial(.,$.$) is the$ Jacobian with respect to position in two dimensions. Some of the geophysically interesting generalizations (flow on a rotating sphere, quasi-geostrophic baroclinic flow, flow over shallow topography, etc.) are included, as in Andrews' paper, by taking $Q$ to be an appropriate potential vorticity. Whenever the function $\Psi(Q)$ is singlevalued-we may speak of 'unifunctional' basic flows-it is found that the finite-amplitude conservation theorem may be expressed solely in terms of Eulerian quantities. But in the more general case of 'multifunctional' basic flows, for which different functions $\Psi(Q)$ apply in different parts of the flow domain, Lagrangian information is needed in order to evaluate the conserved density and its flux for a finite-amplitude disturbance. The amount of Lagrangian information then required is discussed in $\$ 5$. The need to specify Lagrangian information, in what at first sight appears to be a purely Eulerian result, is reminiscent of other cases in which general theorems of analytical mechanics have resisted attempts to express them in terms of Eulerian quantities alone, the case of Hamilton's principle and the 'Lin constraint' being perhaps the best known (e.g. Penfield 1966; Bretherton 1970; Salmon 1982).

The related theoretical work discussed in $\$ \S 6,7$ sets the present results in a wider perspective, and shows how they may be generalized to more complicated fluid systems. It comprises, first, the nonlinear stability theorems of Arnol'd (1965, 1966a) and their recent extensions to other fluid systems by, among others, Holm et al. (1983, 1985) and Abarbanel et al. (1986) - see also the related work of Benjamin (1972) - and, second, the generalized Hamiltonian formulation of incompressible vortex dynamics in the Eulerian description (e.g. Kuznetsov \& Mikhailov 1980; Morrison 1982; Olver 1982; Marsden \& Weinstein 1983; Benjamin 1984, 1986; Marsden 1984; Lewis et al. 1986). The key steps towards this Eulerian Hamiltonian formulation were again taken by Arnol'd (1966, 1969$)$. The resulting body of theory 
not only clarifies where Andrews' wave-activity conservation theorem comes from, and its finite-amplitude counterpart presented here, but also reveals the origin of the (analytically similar) finite-amplitude 'available potential energy' and 'generalized Eliassen-Palm' conservation theorems noted by Holliday \& McIntyre (1981) and Killworth \& McIntyre (1985) from elementary considerations. The latter conservation theorem was used to place rigorous bounds on the wave absorptivity of nonlinear Rossby-wave critical layers; a finite-amplitude theorem was essential for this purpose since the critical layer can be regarded as a narrow region of wave breaking, in the sense already alluded to.

The above-mentioned work on nonlinear stability theorems (see Holm et al. 1985 for a comprehensive review) is noteworthy in that it offers systematic methods for obtaining stability results like Arnol'd's, where they exist, using the 'Casimir invariants' which arise in the generalized Hamiltonian theory (e.g. Schiff 1968, p. 209; Sudarshan \& Mukunda 1974, pp. 321ff.). These methods have already led, for example, to some profoundly interesting answers to longstanding questions about the nonlinear stability of stratified shear flows (Abarbanel et al. 1984, 1986). Moreover, it is straightforward to extend the methods to find exact, local, quasiEulerian conservation relations, of the kind exemplified here, in many other problems involving finite-amplitude disturbances to stable or unstable basic flows. For instance the restriction to quasi-geostrophic motion assumed in Andrews' paper can be lifted (although the details are complicated). The basic procedure is illustrated in $\$ 7$.

The essential ideas leading to all these conservation and stability theorems are contained in Arnol'd's (1966a) paper, in which two fully nonlinear stability theorems are established. It is a curious fact that, until very recently, this paper seems to have been largely overlooked in the Western fluid-mechanical literature. The methods used in Arnol'd's (1966a) paper are not to be confused with the closely related, but less powerful, variational method given in his earlier and more widely quoted 1965 paper, which by itself proves only linear stability albeit pointing clearly towards the finite-amplitude results (Arnol'd 1978, p. 335; Holm et al. 1985).

\section{Vorticity, potential-vorticity and wave-energy equations}

We begin by considering two-dimensional, incompressible flow; the extensions to the spherical and the baroclinic quasi-geostrophic cases are straightforward and are given in Appendices A and B. The model system is governed by conservation of potential vorticity $P$ :

$$
\frac{\mathrm{D} P}{\mathrm{D} t}=0 \text {, }
$$

i.e.

$$
P_{t}+\partial(\Phi, P) \equiv P_{t}-\Phi_{y} P_{x}+\Phi_{x} P_{y}=0,
$$

where $x$ and $y$ are horizontal Cartesian coordinates, $t$ the time, $\Phi$ the stream function, and where we take

$$
P \equiv \nabla^{2} \Phi+f(x, y) \text {. }
$$

Classical two-dimensional, inviscid flow is included as the special case $f=0, P$ then being vorticity in the usual sense. By taking $f$ to be a prescribed function of $x$ and $y$ we obtain one of the usual 'barotropic models' of flow in the atmosphere or oceans, in which $f$ approximately represents the combined effects of variable Coriolis parameter and shallow topography. For remarks about more general models, see Appendices A and B. We may postpone a discussion of the boundary conditions until $\S 6$, since $\$ \S 2-5$ are concerned only with local conservation relations. 
Now consider a steady flow $(\Phi, P)=(\Psi, Q)$, called the 'basic flow', where

$$
Q=\nabla^{2} \Psi+f(x, y) .
$$

$(\Psi, Q)$ is itself a solution to $(2.1)$, so that

$$
\partial(\Psi, Q)=\mathbf{0}
$$

Defining the disturbance $(\psi, q)$, depending on $x, y$ and $t$, by

$$
\Phi \equiv \Psi+\psi, \quad P \equiv Q+q,
$$

so that

$$
q=\nabla^{2} \psi
$$

and substituting (2.5) into (2.1), one obtains the exact equation for the disturbance potential vorticity,

$$
q_{t}+\partial(\psi, Q+q)+\partial(\Psi, q)=0
$$

using (2.4). We shall also need an exact equation for the disturbance energy

$$
\hat{E}=\frac{1}{2}|\boldsymbol{\nabla} \psi|^{2},
$$

which is an $O\left(a^{2}\right)$ quantity as disturbance amplitude $a \rightarrow 0$. To derive the disturbanceenergy equation, multiply (2.7) by $\psi$ and then note that

$$
\psi q_{t} \equiv \psi \nabla^{2} \psi_{t}=\nabla \cdot\left(\psi \nabla \psi_{t}\right)-\frac{\partial}{\partial t} \hat{E},
$$

while

$$
\psi \partial(\psi, Q+q)=\partial\{\psi,(Q+q) \psi\}=\nabla \cdot\{(Q+q) \psi u\}
$$

and

$$
\psi \partial(\Psi, q)=\psi \boldsymbol{\nabla} \cdot(q U)=\boldsymbol{\nabla} \cdot(q \psi U)-q \partial(\Psi, \psi),
$$

where $u$ and $U$ are the velocity fields corresponding to $\psi$ and $\Psi$ respectively, namely

$$
\boldsymbol{u} \equiv(u, v) \equiv\left(-\psi_{y}, \psi_{x}\right) \equiv \hat{\boldsymbol{z}} \times \boldsymbol{\nabla} \psi ; \quad \boldsymbol{U}=\hat{\boldsymbol{z}} \times \boldsymbol{\nabla} \Psi .
$$

Here, by definition, $\hat{z} \times \nabla \equiv(-\partial / \partial y, \partial / \partial x)$. The result can be written in the form

$$
\frac{\partial}{\partial t} \hat{E}+\nabla \cdot\left\{-\psi \nabla \psi_{t}-q \psi(U+u)+\frac{1}{2} \psi^{2} \hat{z} \times \nabla Q\right\}=q \partial(\psi, \Psi),
$$

where the fact that

$$
\boldsymbol{\nabla} \cdot\left\{\frac{1}{2} \psi^{2} \hat{z} \times \nabla Q\right\}=-\nabla \cdot\left\{\frac{1}{2} Q \hat{z} \times \nabla\left(\psi^{2}\right)\right\}=-\nabla \cdot\{Q \psi u\}
$$

has been used. We note that the right-hand side of $(2.9)$ can be manipulated into the form

$$
q \partial(\psi, \Psi)=\frac{\partial}{\partial x_{i}}\left\{\left(u_{i} u_{j}-\frac{1}{2} u_{k} u_{k} \delta_{i j}\right) U_{j}\right\}-\frac{1}{2}\left\{\frac{\partial U_{i}}{\partial x_{j}}+\frac{\partial U_{j}}{\partial x_{i}}\right\} u_{i} u_{j}
$$

(with summation over repeated indices), exhibiting the appropriate form of the Reynolds stress source term for disturbance energy.

If the basic-flow strain rate $\frac{1}{2}\left(\partial U_{i} / \partial x_{j}+\partial U_{j} / \partial x_{i}\right)$ vanishes, then the disturbance energy density $E$ becomes a conserved density, with flux given by the difference between the quantities within braces whose divergences appear in (2.9) and (2.11), viz.

$$
-q \psi(U+u)-\psi \frac{\partial}{\partial t} \nabla \psi+\frac{1}{2} \psi^{2} \hat{z} \times \nabla Q-\left(u u-\frac{1}{2}|u|^{2} \boldsymbol{I}\right) \cdot U
$$

where $I$ is the two-dimensional identity tensor. In the case of zero basic-flow strain rate this last expression may also be written as

$$
\mathscr{E}(U+u)-\psi \frac{\mathrm{D}}{\mathrm{D} t} \nabla \psi+\frac{1}{2} \psi^{2} \hat{z} \times \nabla Q
$$


plus an identically non-divergent term. When the basic flow has weak shear and nearly constant $\nabla Q$, then $\hat{E}$ is approximately conserved, and its flux as given by either of $(2.12 a, b)$ can be shown to have the 'group-velocity property' for a slowly varying train of purely progressive Rossby waves. By the group-velocity property we mean that the wavelength or period average of the flux (2.12) divided by the corresponding average of $\hat{E}$ is equal to the group velocity of the waves, to a first approximation in which $U, \nabla Q$, and wave amplitude are assumed constant. The alternative form of the flux suggested by (2.10), in which $-Q \psi u$ replaces $\frac{1}{2} \psi^{2} \hat{z} \times \nabla Q$, does not possess the group-velocity property (cf. Longuet-Higgins 1964; Pedlosky 1979, \$§3.21, 6.10), a fact which is related to the generally large magnitude of $Q$, and the tendency of $\psi$ and $\boldsymbol{u}$ to be nearly out of phase, in an almost-plane Rossby wave.

\section{A finite-amplitude conservation theorem}

In order to obtain an exact conservation theorem when the basic-flow strain rate $\frac{1}{2}\left(\partial U_{i} / \partial x_{j}+\partial U_{j} / \partial x_{i}\right)$ is not zero, it suffices to turn the right-hand side of (2.9) into a term of the form $\mathrm{D}(\mathrm{)} / \mathrm{D} t$. This is done by introducing the functional dependence of $\Psi$ on $Q$ implied by (2.4):

$$
\boldsymbol{\Psi}=\boldsymbol{\Psi}(Q) \text {. }
$$

We assume at first that $\Psi$ is a single-valued function of $Q$. Consider the $O\left(a^{2}\right)$ function

$$
B(Q, q) \equiv \int_{0}^{q}\{\Psi(Q+\tilde{q})-\Psi(Q)\} \mathrm{d} \tilde{q}
$$

where $Q$ is held constant as the integration with respect to $\tilde{q}$ is carried out. This is of quadratic order for small disturbance amplitude. The following two properties are easily established from (3.2):

$$
\frac{\partial B}{\partial Q}=\Psi(Q+q)-\Psi(Q)-q \Psi^{\prime}(Q)
$$

where $\Psi^{\prime}(Q)$ denotes the derivative of $\Psi(Q)$, and

$$
\frac{\partial B}{\partial q}=\Psi(Q+q)-\Psi(Q)
$$

Also (2.1), (2.5) and (2.7) yield

$$
\frac{\mathrm{D} Q}{\mathrm{D} t}=-\frac{\mathrm{D} q}{\mathrm{D} t}=\partial(\psi, Q)
$$

Then (3.3)-(3.5) together imply that

$$
\frac{\mathrm{D}}{\mathrm{D} t} B(Q, q)=\frac{\partial B}{\partial Q} \frac{\mathrm{D} Q}{\mathrm{D} t}+\frac{\partial B}{\partial q} \frac{\mathrm{D} q}{\mathrm{D} t}=-q \Psi^{\prime}(Q) \partial(\psi, Q)=-q \partial(\psi, \Psi),
$$

which is the form required. The finite-amplitude conservation theorem then follows immediately from (2.9) and (3.6):

$$
\frac{\partial}{\partial t} A+\nabla \cdot F=0
$$

where

$$
A=\hat{E}(x, y, t)+B\{Q(x, y), q(x, y, t)\},
$$

and

$$
\boldsymbol{F}=\{B(Q, q)-q \psi\}(\boldsymbol{U}+\boldsymbol{u})-\psi \frac{\partial}{\partial t} \boldsymbol{\nabla} \psi+\frac{1}{2} \psi^{2} \hat{z} \times \nabla Q
$$

The device (3.2) was used by Arnol'd in his little-quoted second paper on stability (Arnol'd 1966a) as a preliminary to proving the two nonlinear stability theorems to 
be discussed in $\$ 6$ below. It was rediscovered by Holliday \& McIntyre (1981) in a different context, that of constructing a finite-amplitude available-potential-energy theorem for incompressible, stratified flow, and used by Killworth \& McIntyre (1985) to derive the finite-amplitude 'generalized Eliassen-Palm' conservation theorem mentioned in the Introduction, which applies to disturbances on a translationally invariant basic state (see (7.11) below). We shall refer to $A$ as (the density of) Arnol'd's invariant.

The form (3.8b) of the conserved flux $F$ is not the only one possible. For instance a few lines of manipulation show that we may use in place of $(3.8 b)$ the expression

$$
A(\boldsymbol{U}+\boldsymbol{u})-\psi \frac{\mathrm{D}}{\mathrm{D} t} \boldsymbol{\nabla} \psi+{ }_{2}^{1} \psi^{2} \hat{z} \times \nabla Q+\frac{1}{2} \partial(\psi, \Psi) \nabla \psi+\frac{1}{2} \psi(\nabla \psi \cdot \nabla U-\nabla \Psi \cdot \nabla u),
$$

which is closer to $(2.12 b)$ and which is equal to $(3.8 b)$ plus the identically nondivergent term $\hat{z} \times \nabla\left(\psi \hat{E}+\frac{1}{2} \psi U \cdot u\right)$. Or again, we may take the flux to be

$$
\{B(Q, q)-q \psi\}(U+u)-\psi \frac{\partial}{\partial t} \nabla \psi-Q \psi u,
$$

which is obtained by subtracting from $(3.8 b)$ the identically non-divergent quantity $\hat{z} \times \nabla\left(\frac{1}{2} Q \psi^{2}\right)$ or by using (2.10). More generally, (3.7) remains true if we add the divergence of any vector field to $A$ and subtract its time derivative from $F$. The forms $(3.8 b, c)$ are preferable to $(3.8 d)$ for some purposes since of the three forms only the first two possess the group-velocity property for a slowly varying Rossby wavetrain, as can easily be verified. They do so, in fact, even for finite-amplitude progressive wavetrains, both in the infinite-plane-wave and in the zonal channel cases, for which the basic sinusoidal solutions are exact. In the channel case one requires $\nabla Q$ constant and transverse to the channel, $U$ constant and along the channel, and $\partial \psi / \partial x=0$ on the boundaries $y=$ constant; the group-velocity property applies to the double average across the channel and over a wavelength or period. It should be cautioned that the averaged values of $A$ and $F$ both vanish if the plane or channel waves are stationary (Plumb 1985 $a$ ). The conservation theorem is still true for a steady disturbance to a steady basic flow, but in that case appears incapable of conveying information about the propagation of (free) Rossby waves.

For unifunctional basic flows (i.e. flows such that $\Psi(Q)$ is single-valued), the function $B(Q,$.$) , and therefore the conserved density A$, are uniquely defined in terms of the properties of the basic flow, since the integral in (3.2) is then determinate. One may then regard the inviscid conservation theorem (3.7) as an Eulerian result, in the sense that the conserved density $A$ and its flux $F$ can each be evaluated at any given point $x$ and time $t$, with no explicit reference to the positions, at earlier times, of the fluid element now at $\boldsymbol{x}$ in the disturbed flow. For multifunctional basic flows, by contrast, there is no means of determining which branch or branches of $\Psi(Q)$ should be used in (3.2) unless Lagrangian information is available. In $\$ 5$ we discuss how (3.2) is then to be interpreted, and how much Lagrangian information is then required.

It might be asked why we do not simply use the difference

$$
\Delta E=\frac{1}{2}|\nabla(\Psi+\psi)|^{2}-\frac{1}{2}|\nabla \Psi|^{2}
$$

between the densities of total energy and basic-flow energy, rather than $A$, as our conserved density. The difference energy $\Delta E$ is equally a conserved density in the situation considered. One reason is that in some applications - such as quantifying wave propagation - it is useful to have a conserved density and flux which are second-order 'wave properties' or 'disturbance properties' in the sense that they can 
be evaluated correct to $O\left(a^{2}\right)$ from a solution calculated on the basis of linearized theory, and so on to higher orders in disturbance amplitude $a$. (For example, this is a necessary condition for the group-velocity property to hold.) $B, A$ and $F$ are evidently wave properties in this sense, for smooth $\Psi(Q)$, whereas $\Delta E$ is not. To evaluate $\Delta E$ even to leading order the disturbance $\psi$ must be known correct to $O\left(a^{2}\right)$.

A further reason why $A$ can be more useful than $\Delta E$ is that its integral over the flow domain may in certain cases have definite sign for arbitrary disturbances, unlike the corresponding integral of $\Delta E$ when $U \neq 0$. For instance inspection of (3.2) and (3.8a) shows that the sign is definite when $\Psi^{\prime}(Q)$ is positive everywhere; this is the basis of one of Arnol'd's two stability theorems (\$6). A conservable density of definite sign, if such exists, may also be useful in problems of wave propagation, since its spatial integral can then be regarded as a straightforward measure of the "amount of wave activity'.

In $\$ 7$ we show that, in a certain sense, the construction of conservable wave properties like $A$ is a unique construction. In the process, the relationship between $(3.8 a)$ and (3.9) is clarified, together with the origin of expressions like (3.2).

\section{The small-amplitude limit}

To connect the finite-amplitude conservation theorem (3.7) with the smallamplitude theorem found by Andrews (1983), consider the limit of small $q$ appropriate to linearized disturbance theory, assuming that $\Psi(Q)$ is smooth. Then (3.2) becomes, correct to leading order,

$$
B(Q, q) \approx \int_{0}^{q} \Psi^{\prime}(Q) \tilde{q} \mathrm{~d} \tilde{q}=\frac{1}{2} \Psi^{\prime}(Q) q^{2}
$$

This confirms that $B$ and therefore $A$ are second-order disturbance properties, in the sense already explained. Their positive definiteness when the derivative $\Psi^{\prime}(Q)$ is positive will again be noted; in such cases $B$ might be thought of as a kind of 'generalized enstrophy'. (In practice it may need to be sharply distinguished from the ordinary enstrophy $\frac{1}{2} q^{2}$, since $\Psi^{\prime}(Q)$ can exhibit strong spatial inhomogeneity across naturally occurring flows, e.g. Hoskins, McIntyre \& Robertson 1985 and references therein.)

Note also that even if $\Psi(Q)$ is multivalued, there is now no ambiguity regarding which branch of the function $\Psi(Q)$ to choose : the value of $\Psi^{\prime}(Q)$ on the right-hand side of (4.1) must evidently be taken as the value belonging to the local streamline of the undisturbed flow. This is meaningful in the context of linearized theory, which assumes inter alia that fluid particles remain close to the basic-flow streamline from which they originated, and have therefore not crossed between regions of the basic flow corresponding to different branches of $\Psi(Q)$. Of course no such assumption is possible in the finite-amplitude case.

In the limit of small $q$, the finite-amplitude velocity field $(U+u)$ must be replaced by its small-amplitude approximation $U$ in the expression $(3.8 b)$ for the conserved flux $F$. Following Andrews, we define the quantity

$$
\Lambda(\Psi) \equiv \frac{\mathrm{d} Q}{\mathrm{~d} \Psi}=\frac{1}{\Psi^{\prime}(Q)}
$$

Then substitution of (4.1) into the linearized form of $(3.8 a, b)$ yields

$$
A \approx \hat{E}+\frac{q^{2}}{2 \Lambda}, \quad F \approx \frac{1}{2 \Lambda}(q-\Lambda \psi)^{2} U-\psi \frac{\partial}{\partial t} \nabla \psi
$$


(3.7) with $(4.3 a, b)$ inserted is the two-dimensional equivalent of Andrews' (1983) small-amplitude conservation theorem. The three-dimensional, quasi-geostrophic version is noted in Appendix B. Alternatively, from (3.8c),

$$
\boldsymbol{F}-\boldsymbol{A U} \approx-\psi \frac{\mathrm{D}_{\mathrm{b}}}{\mathrm{D} t} \boldsymbol{\nabla} \psi+\frac{1}{2} \psi^{2} \hat{z} \times \nabla Q+\frac{1}{2} \partial(\psi, \Psi) \nabla \psi+\frac{1}{2} \psi(\nabla \psi \cdot \nabla U-\nabla \Psi \cdot \nabla u),
$$

where $\mathrm{D}_{\mathrm{b}} / \mathrm{D} t \equiv \partial / \partial t+\boldsymbol{U} \cdot \boldsymbol{\nabla}$. For a slowly varying Rossby wavetrain the right-hand side of $(4.3 c)$ is equal to $A$ times the intrinsic group velocity, after suitable averaging.

\section{Multifunctional basic flows}

How is the integral in (3.2) to be interpreted in the general case of a finite-amplitude disturbance to a multifunctional basic flow? To take a simple example, consider a flow parallel to the $x$-axis with velocity profile

and vorticity profile

$$
U(y)=\sinh y+\epsilon \log \cosh y
$$

$$
Q(y)=-\cosh y-\epsilon \tanh y
$$

(taking $f=0$ ). The resulting $\Psi(Q)$-curve folds back on itself in the manner illustrated in figure $1(a)$, which shows the case $\epsilon=0.5$ (heavy curve). Figure $1(b)$ depicts the corresponding velocity profile. The flow is multifunctional, $\Psi(Q)$ having two branches, except when $\epsilon=0$. A more complicated example, with non-zero $f$, will be noted shortly (figure 2 below).

Multifunctionality implies that knowledge of the values of $Q$ and $q$ is insufficient to evaluate the integral in (3.2). In the case of figure 1(a), for instance, specifying the values of $Q$ and $q$ permits two choices for each limit of integration. The ambiguity would be resolved if the relevant segment, $a b$ say, of the $\Psi(Q)$-curve were specified, where $a$ corresponds to the lower limit of integration and $b$ to the upper limit. How, then, do we choose $a$ and $b$ such that the conservation law (3.7) always holds?

A sufficient answer is to make $a$ fixed for an Eulerian observer and $b$ fixed for a Lagrangian observer. More precisely,

(i) the point $a$ is chosen to be $(\Psi, Q)$ where $(\Psi, Q)$ are the background values at the current position $x$ of a given fluid element; and

(ii) the point $b$ is chosen such that it remains fixed in the $(\Psi, Q)$-plane whenever we follow the motion of the fluid element (so that $Q+q$ is constant).

These two conditions ensure that the intermediate steps (3.3)-(3.6) in the derivation of (3.7) make mathematical sense. The appropriateness of condition (ii), in particular, can be seen at once from continuity considerations. In order for (3.7) to hold true, the value of $B$, as defined by the integral in (3.2), must have a material rate of change which always agrees with the right-hand side of (3.6) as a fluid element moves across the background of undisturbed streamlines, making the point $a$ move along the $\Psi(Q)$-curve. The point $b$ cannot be allowed to jump discontinuously from branch to branch of the $\Psi(Q)$-curve as the fluid element moves; the resulting jump in the value of $B$ would generally contradict (3.6).

Once the segment $a b$ is specified, the value of $B$ is unambiguously defined. In the case illustrated in figure $1(a)$, for instance, $B$ is equal to the vertically hatched area minus the horizontally hatched area. The general rule, applicable also to more complicated cases like that of figure 2 below, is as follows. Drop a perpendicular from $b$ to the horizontal ( $\Psi=$ constant) line through $a$, meeting it at the point $c$ say; the 


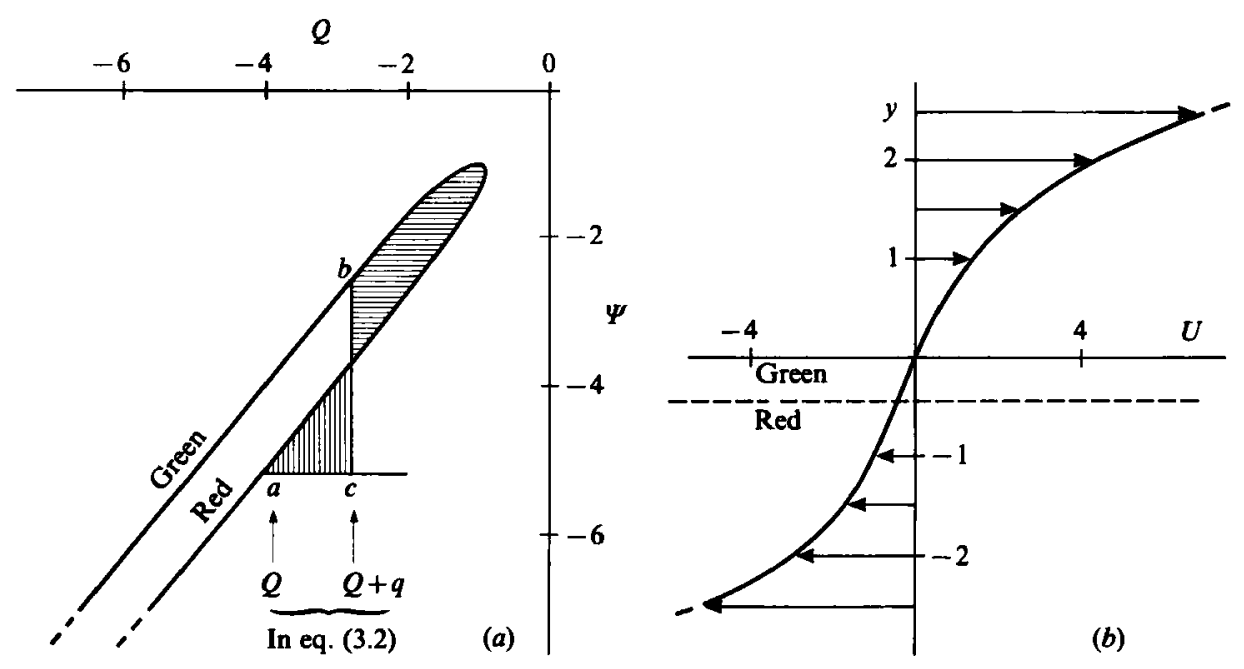

FIGURE 1. (a) The function $\Psi(Q)$ for the velocity and vorticity profiles given by (5.1) and (5.2), with $\epsilon=0.5 ;(b)$ the corresponding basic velocity profile. The vertically hatched area in $(a)$ represents a positive contribution to the integral in (3.2), and the horizontally hatched area a negative contribution. The dashed line in (b) divides the domain of the basic flow into the two parts which correspond to the two branches of the $\Psi(Q)$-curve. The 'colour coding' indicates which parts of the velocity profile belong to which parts of the $\Psi(Q)$-curve. (Note that the stream function sign convention is the geophysical one with $U=-\partial \Psi / \partial y, V=\partial \Psi / \partial x$.)

value of the integral in (3.2) is taken as the area within the closed curve abca, counting as positive those parts lying to the right of the relevant segment $a b$ of the $\Psi(Q)$-curve, and as negative those lying to the left, looking from $a$ towards $b$.

Condition (ii) tells us not only that Lagrangian information is needed in order to make $B$ determinate, but also how much Lagrangian information. In the example of figure 1, for instance, each fluid element must be tagged with just one 'bit' of information, namely on which of the two branches to place the point $b$. If we wish, we may think of some of the fluid as coloured red, and some green, where red denotes the lower branch of the $\Psi(Q)$-curve and green the upper branch, as indicated in figure 1. Then the essential requirement is to keep track of where the red and green fluid goes as the motion evolves, and in particular to keep track of the moving boundary between the two colours. Although this represents far less information than a full Lagrangian specification of the fluid motion, it is by no means a trivial amount. The pattern of red and green can get very complicated, the red and green fluid becoming more and more intimately intertwined, into spiral and other highly convoluted patterns, in the familiar way characteristic of unsteady, nonlinear, vortical disturbances (such as may be associated with breaking Rossby waves). A notation which explicitly recognizes what $B$ depends on might be

$$
B=B(Q, q, \Psi, \text { colour }),
$$

although it should be cautioned that not all the 'arguments' are independent. The third argument is needed only to enforce condition (i) (and in place of $\Psi$ one could equally well write $\boldsymbol{x}$ ). The last argument, enforcing condition (ii), may be thought of as the colour of the undisturbed streamline from which the fluid element originated. It should be noted that this entails a tacit assumption (which is logically unnecessary but which it is often natural to make) that the disturbance is dynamically 'realizable' in the sense that it could, in fact, have evolved from the undisturbed basic state. 


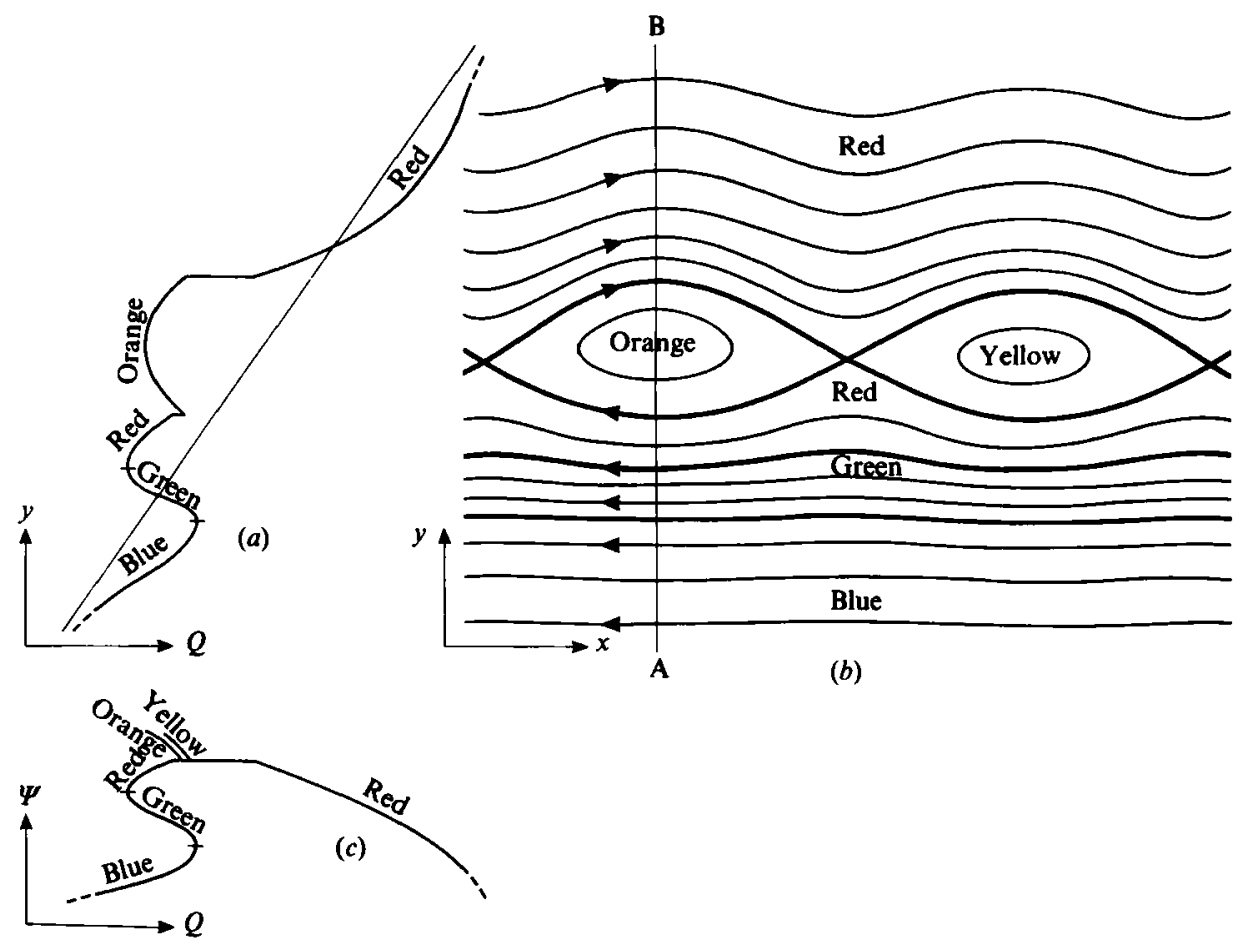

Figure 2. $Q(y), \Psi(x, y)$ and $\Psi(Q)$ for a more complicated multifunctional basic flow (schematic). Corresponding portions of each sketch are indicated by the colours. The profile of $Q(y)$ at top left is taken along the section $\mathrm{AB}$ through the streamline pattern shown above; the sloping line on the left shows $Q(y)$ in the absence of flow. $\Psi(Q)$ is the multivalued function shown at bottom left. The fact that all five colours are needed to distinguish different streamlines with the same value of $Q$ can be seen by considering for instance the value of $Q$ for the tip of the yellow 'antenna' on the $\Psi(Q)$-curve.

If the $\Psi(Q)$-curve has $n$ branches, then we need $n$ colours. A multifunctional, non-parallel basic flow requiring five colours is sketched in figure 2 . This case has non-zero $f=f(y)$, indicated by the sloping straight line in figure $2(a)$. In the basic-flow streamline pattern on the right, the boundaries between regions of differing colour are marked by the heavy streamlines. The graph on the left shows the profile of $Q$ on a line $x=$ constant, marked $\mathrm{AB}$, passing through the left-hand region of closed streamlines. The $\Psi(Q)$-curve is placed at bottom left in order to facilitate comparison of $Q$-values by eye. The reader is invited to colour in the basic-flow streamline pattern, and the $\Psi(Q)$-curve, as indicated, and to imagine what might happen to the shapes of the boundaries between the colours if a disturbance were introduced. Apart from the green and blue regions, which were put in to show some of the theoretical possibilities (and which would make the flow extremely unstable), this corresponds to a type of basic flow of potential interest in connection with theoretical ' wave-mean' models of large-scale atmospheric circulations, as explained for instance by Andrews $(1983)$ and Plumb $(1985 a, b)$. The orange and yellow regions represent subtropical anticyclones. The $\Psi(Q)$-curve at the bottom left of figure 2 is simply connected (as is true in most examples of practical interest), and the integral (3.2) can still be rendered unambiguous, therefore, in the way described above, provided only that we keep track of the whereabouts of all five colours so that the position of the point $b$ on the $\Psi(Q)$-curve is always known for any given $x$. 
We now turn to the wider theoretical background, beginning with a review of the related stability concepts.

\section{Stability and instability}

In this section we restrict attention, as did Arnol'd $(1965,1966 a)$, to unifunctional basic flows. Consider the integral of $A$ over the flow domain $D$,

$$
\mathscr{A} \equiv \iint_{D} A \mathrm{~d} x \mathrm{~d} y=\iint_{D}(\hat{E}+B(Q, q)) \mathrm{d} x \mathrm{~d} y .
$$

The conservation relation (3.7) implies that

$$
\frac{\mathrm{d} \mathscr{A}}{\mathrm{d} t}=0
$$

whenever conditions at the boundary $\partial D$ are such that

$$
\oint_{\partial D} \boldsymbol{F} \cdot \hat{\boldsymbol{n}} \mathrm{d} s=\mathbf{0}
$$

where $s$ is arclength along $\partial D$ and $\hat{n}$ is the unit outward normal to $\partial D$. The exact result (6.2) was noted as a lemma in Arnol'd's second stability paper (1966a), where it was derived by a slightly different route.

It is clear that in unifunctional cases where $\Psi^{\prime}(Q)$, and therefore $B, A$ and $\mathscr{A}$, are positive definite, (6.2) has implications for the finite-amplitude stability of the basic flow. For instance Arnol'd (1966a) used (6.2), together with the hypothesis that $\Psi^{\prime}(Q)$ is positive, finite and bounded away from zero, (6.9) below, to prove a stability theorem applicable to disturbances of arbitrary amplitude. This is to be sharply distinguished from the more widely quoted result presented in Arnol'd's earlier (1965) paper, the proof of which was for small amplitude only (Arnol'd 1978, p. 335; Holm et al. 1985).

Arnol'd further noted that in certain circumstances $\mathscr{A}$ can be negative definite, and proved a second stability theorem in that case, likewise applicable to disturbances of arbitrary amplitude. As will be explained, this second theorem is related to the suppression of certain inviscid instabilities by boundary constraints. They include ordinary shear instabilities, meaning those dependent on the existence of a nonmonotonic cross-stream $Q$ gradient, plus at least one kind of instability which can operate in certain non-parallel flows with monotonic cross-stream $Q$ gradients. The first clear theoretical characterizations of this latter instability appear to have been given independently by Charney \& DeVore (1979) and by Plumb (1979), with adumbrations going back to papers by Matsuno \& Hirota (1966) and Hirota (1967). It may generally be called 'self-tuning resonant instability', for reasons to be explained, and cases of it have been studied in the recent meteorological literature under the headings 'topographic instability', 'orographic instability' and 'form-drag instability', in connection with hypotheses about certain low-frequency fluctuations of the large-scale flow in the Earth's atmosphere.

The boundary condition (6.3) is important to what follows, and so we digress to examine it a little more closely before proceeding. It is automatically satisfied, of course, in doubly periodic rectangles, or in infinite domains in which $|\boldsymbol{F}|$ decreases more rapidly than the inverse of distance. For the less obvious case of an enclosed domain $D$ at whose boundary $\partial D$ the normal components of $U$ and $u$ vanish, we have

$$
\frac{\partial \psi}{\partial s}=0 \quad \text { on } \partial D
$$




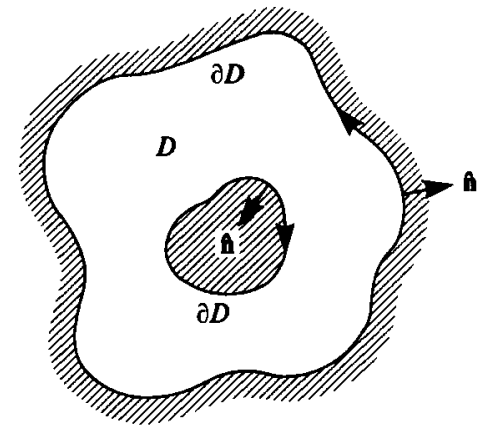

Fiavre 3. Sketch of a hypothetical doubly connected domain $D$, showing the orientations of arclength and normal assumed in the text. Inviscid, steady flow around such an annular domain can be unstable even for monotonic cross-stream $Q$ profiles, because of the self-tuning resonant instability mechanism.

In that case (6.3) is equivalent to

$$
\oint_{\partial D} \psi \frac{\partial}{\partial t} \frac{\partial \psi}{\partial n} \mathrm{~d} s=\psi \frac{\mathrm{d}}{\mathrm{d} t} \oint_{\partial D} \boldsymbol{u} \cdot \mathrm{d} \boldsymbol{s}=\mathbf{0}
$$

Here (2.10) has been used to show that the normal component of the term $\frac{1}{2} \psi^{2} \hat{z} \times \nabla Q$ appearing in the definition $(3.8 b, c)$ of $F$ integrates to zero, leaving only the term involving $\partial \psi / \partial t$. Alternatively, we may use (3.8d). The condition (6.5) holds true, by Kelvin's circulation theorem, for the inviscid, free motions under consideration. If the domain $D$ is multiply connected, as illustrated in figure 3, then the same consideration shows that (6.5) holds separately on each connected portion of $\partial D$. One can, without loss of generality, impose the stronger boundary condition

$$
\oint_{\partial D} u \cdot \mathrm{d} s=\oint_{\partial D} \frac{\partial \psi}{\partial n} \mathrm{~d} s=0
$$

on each connected portion of $\partial D$, redefining the basic flow if necessary so as to absorb into it any non-zero (and constant) disturbance boundary circulations $\oint \boldsymbol{u} \cdot \mathrm{d} s$. In that case we have

$$
\iint_{D} q \mathrm{~d} x \mathrm{~d} y=0
$$

recalling that $q=\nabla^{2} \psi$. Note that (6.6) is automatically true of any disturbance which is dynamically 'realizable' in the sense of $\$ 5$, i.e. which could have developed from a state of zero disturbance at $t=-\infty$. It is to such disturbances that Arnol'd's stability theorems apply.

It should be carefully noted that when $D$ is multiply connected $\psi$ need not be zero, nor time-independent, on each separate portion of $\partial D$. For example the self-tuning resonant instability, which can manifest itself in annular domain geometries such as that shown in figure 3, generally gives rise to a disturbance for which (6.4) and (6.6) are satisfied on each wall but in which, at small disturbance amplitude, there is an exponentially growing difference $\Delta \psi$ between the values of $\psi$ on the two walls. This represents a change in the mass flow around the annulus, induced by the growing disturbance potential-vorticity field. The associated angular momentum change, which is generally non-zero, is supplied through pressure forces on the wavy 
boundary. Such angular momentum changes are to be expected from the nature of the self-tuning resonant instability mechanism. As Plumb (1981) explains, that mechanism involves the excitation of forced, stationary Rossby waves by the boundary (or by anything else that forces the flow to be non-axisymmetric), and a tuning towards resonance brought about by the wave-induced changes in the flow around the annulus.

The remainder of this section discusses what can be said about stability on the basis of (6.2); see also Holm et al. (1985). We can distinguish at least three useful senses in which a unifunctional basic flow may be said to be nonlinearly stable when $\mathscr{A}$ is positive or negative definite. None of them implies that interesting disturbance phenomena cannot occur and have important effects (e.g. figure 4 below), but each restricts the growth of a wide class of disturbances.

The first sense is simply that temporal constancy, and therefore boundedness, of the definite functional $\mathscr{A}$ can itself be regarded as a statement about nonlinear stability, in the practical sense that it rules out most of the growing disturbances considered in instability theories - for example any temporally growing disturbance representable as a finite sum of terms each having a prescribed spatial form, such as is often assumed in weakly nonlinear theories. Linear stability (to exponentially growing normal-mode disturbances) is implied a fortiori. ( $A$ might appropriately be called a Liapunov functional, by analogy with finite-dimensional systems; cf. Hirsch \& Smale 1974, §9.3.)

The second sense is that used in the general functional-analytic theory of dynamical systems. In that theory stability is usually taken to entail boundedness of some norm \|\| measuring the size of the disturbance $\psi$. More precisely, stability in this second sense, often referred to as 'nonlinear stability' or 'Liapunov stability', means that given any number $\epsilon>0$, there exists a number $\delta>0$ such that

$$
\|\psi\|<\delta \quad \text { at } t=0 \Rightarrow\|\psi\|<\epsilon \quad \text { for all } t
$$

This definition of nonlinear stability, whose meaning depends on the particular choice of norm, is natural for mathematical purposes because of the central role of normed vector spaces in functional-analytic theory. It is the definition with which Arnol'd was primarily concerned. We recall that a norm must, first of all, be a metric - i.e. it must be non-negative, non-zero for any non-zero function $\psi$, unaffected by multiplying the function by -1 , and must satisfy the triangle inequality $\left\|\psi_{1}+\psi_{2}\right\| \leqslant\left\|\psi_{1}\right\|+\left\|\psi_{2}\right\|$. Second, it must also be homogeneous in the sense that $\|c \psi\|=|c|\|\psi\|$ for any constant $c$. The square root of the functional $\mathscr{A}$ evidently fails in general to qualify as a norm, or even as a metric (in contrast with the square root of the integral of $(4.3 a)$, the $O\left(a^{2}\right)$ approximation to $\mathscr{A}$, which qualifies as both $\left.\dagger\right)$. The only exception (at finite amplitude) is the case where $\Psi^{\prime}(Q)$ is constant so that (4.1) is exact; examples are given in (6.29) and (6.31) below. It is clear that in order to prove stability in the second, Liapunov sense we must construct inequalities that throw away some of the information contained in (6.2), thus restricting the growth of a given disturbance less sharply than before.

Liapunov's theorem guarantees that stability in the first sense implies stability in the second sense for finite-dimensional systems (e.g. Hirsch \& Smale 1974). It is

$\dagger$ Ball \& Marsden (1984) give an example from elasticity theory which illustrates the dangers inherent in a naive use of $O\left(a^{2}\right)$ approximations like $(4.3 a)$ in discussions of stability in infinitedimensional systems. 
because we are dealing with infinite-dimensional systems that we need to distinguish between the two senses.

There is a third sense of stability which includes, but is stronger than, the second sense, and which is also relevant here albeit not often discussed. In some problems one not only has Liapunov stability - that is, given any $\epsilon$, some $\delta$ can be found such that (6.8) holds - but one may also have that, given any $\delta$, some $\epsilon$ can always be found such that $(6.8)$ holds. Stability in this third sense implies that the disturbance can always be bounded, no matter how large it may be initially (the bound going to zero as the initial disturbance amplitude goes to zero). An elementary example of stability in the second but not the third sense is the case of a particle in static equilibrium in a shallow potential well on a hilltop. To be stable in the third sense the particle would have to be contained in an infinitely deep potential well.

Arnol'd's two theorems actually prove stability in both the second and third senses, as we now show. The hypothesis required by the first theorem is that the basic flow be unifunctional and satisfy

$$
0<c \leqslant \Psi^{\prime}(Q) \leqslant C<\infty .
$$

Putting this together with (6.2) and (3.8a) we get the following a priori estimate for all $t$ (Arnol'd 1966a, theorem 1):

$$
\iint_{D}\left(|\nabla \psi|^{2}+c\left(\nabla^{2} \psi\right)^{2}\right) \mathrm{d} x \mathrm{~d} y \leqslant 2 \mathscr{A} \leqslant \iint_{D}\left(\left|\nabla \psi_{0}\right|^{2}+C\left(\nabla^{2} \psi_{0}\right)^{2}\right) \mathrm{d} x \mathrm{~d} y
$$

where $\psi_{0} \equiv \psi_{t-0}$. Define

$$
\|\psi\|_{+} \equiv\left\{\iint_{D}\left(|\nabla \psi|^{2}+c\left(\nabla^{2} \psi\right)^{2}\right) \mathrm{d} x \mathrm{~d} y\right\}^{\frac{1}{2}} \geqslant 0 .
$$

Because $c$ is positive and independent of $\psi$, and because the square root is taken, this qualifies as a norm. To prove stability under the hypothesis $(6.9)$, given any $\epsilon>0$ choose

$$
\delta^{2}=c \epsilon^{2} / C
$$

Then if $\left\|\psi_{0}\right\|_{+}<\delta,(6.10)$ implies that

$$
\begin{aligned}
\|\psi\|_{+}^{2} & =\iint_{D}\left(|\nabla \psi|^{2}+c\left(\nabla^{2} \psi\right)^{2}\right) \mathrm{d} x \mathrm{~d} y \leqslant \iint_{D}\left(\left|\nabla \psi_{0}\right|^{2}+C\left(\nabla^{2} \psi_{0}\right)^{2}\right) \mathrm{d} x \mathrm{~d} y \\
& \leqslant \frac{C}{c} \iint_{D}\left(\left|\nabla \psi_{0}\right|^{2}+c\left(\nabla^{2} \psi_{0}\right)^{2}\right) \mathrm{d} x \mathrm{~d} y=\frac{C}{c}\left\|\psi_{0}\right\|_{+}^{2}<\epsilon^{2} .
\end{aligned}
$$

This proves nonlinear stability both in the second (Liapunov) and in the third, stronger sense, with $\epsilon$ given in terms of $\delta$ by $\epsilon^{2}=C \delta^{2} / c$, and provides a bound on the norm of a disturbance of any initial amplitude whatever.

We note in passing that stability in both the second and third senses holds with respect to the norm defined by taking $C$ rather than $c$ in (6.11). The proof is essentially the same, with a slight re-ordering of the inequalities and with $\epsilon^{2} / \delta^{2}$ again taking the value $C / c$. Other variants are possible.

Now consider the negative definite case. If the basic flow is unifunctional and if positive constants $c, C$ exist such that

$$
0<c \leqslant-\Psi^{\prime}(Q) \leqslant C<\infty,
$$

then (6.10) is replaced by the a priori estimate

$$
\iint_{D}\left(c\left(\nabla^{2} \psi\right)^{2}-|\nabla \psi|^{2}\right) \mathrm{d} x \mathrm{~d} y \leqslant-2 \mathscr{A} \leqslant \iint_{D}\left(C\left(\nabla^{2} \psi_{0}\right)^{2}-\left|\nabla \psi_{0}\right|^{2}\right) \mathrm{d} x \mathrm{~d} y
$$


(Arnol'd 1966a, theorem 2). If moreover the functional

$$
\iint_{D}\left(|\nabla \psi|^{2}-c\left(\nabla^{2} \psi\right)^{2}\right) \mathrm{d} x \mathrm{~d} y
$$

is negative definite, for the given domain and boundary conditions, then both the right- and left-hand sides of (6.15) are positive definite and we can again make deductions about stability (Arnol'd 1966a). Stability in both the second and third senses is again implied, as we show next.

There are two points to be noted about (6.15) and (6.16) which make this case slightly less straightforward than the positive definite case (and which Arnol'd did not explain in great detail). First, in order for either $\mathscr{A}$ or (6.16) to be negative definite, for given $\Psi(Q)$, it is necessary that the domain $D$ be small enough, in one or more directions, to restrict the scale of the disturbance. In the case of (6.16) this can be made precise as follows. If we define the scale of the disturbance to be $\kappa_{\mathrm{m}}^{-1}>0$, where

$$
\left\{\kappa_{\mathrm{m}}(\psi)\right\}^{2} \equiv \frac{\iint\left(\nabla^{2} \psi\right)^{2} \mathrm{~d} x \mathrm{~d} y}{\iint|\nabla \psi|^{2} \mathrm{~d} x \mathrm{~d} y}
$$

and if $\psi$ satisfies the boundary conditions $(6.4)$ and $(6.6)$ - here it is essential to use (6.6) and not (6.5) - then we have the Poincaré inequality

$$
\kappa_{\mathrm{m}} \geqslant k_{0},
$$

where $k_{0}$ is a positive number whose square is the lowest non-trivial eigenvalue of the problem posed by the equation

$$
\nabla^{2} \psi+k^{2} \psi=0
$$

in a given domain, together with the boundary conditions (6.4) and (6.6). The inequality (6.18) can be obtained by expanding $\psi$ and $\nabla^{2} \psi$ in terms of the eigenfunctions of the boundary-value problem just stated and then substituting the expansions into $(6.17)$, after using the relation

$$
\iint_{D}|\nabla \psi|^{2} \mathrm{~d} x \mathrm{~d} y=-\iint_{D} \psi \nabla^{2} \psi \mathrm{d} x \mathrm{~d} y,
$$

which again depends on the boundary conditions (6.4) and (6.6). From (6.17) and (6.18),

$$
\iint_{D}\left(\nabla^{2} \psi\right)^{2} \mathrm{~d} x \mathrm{~d} y \geqslant k_{0}^{2} \iint_{D}|\nabla \psi|^{2} \mathrm{~d} x \mathrm{~d} y .
$$

This shows that the hypothesis required to make (6.16) negative definite is just

$$
c k_{0}^{2}>1 \text {. }
$$

The hypothesis is necessary, as well as sufficient, because equality holds in (6.21) in the case where $\psi$ is proportional to the lowest non-trivial eigenfunction, as can be seen by using (6.20) and then (6.19). For given $c$ this makes it essential that the boundaries restrict the size of the domain $D$ in at least one direction: if the boundaries were to recede to infinity in all directions, then $k_{0}$ would tend to zero.

The second point about (6.15) and (6.16) is that, even after negative definiteness of (6.16) is established, stability in the second or third senses will not follow from a simple majorant argument like (6.13). In manipulating the inequalities we must make further use of the hypothesis (6.22) and the Poincaré inequality, (6.18) or 
(6.21). Following Arnol'd (1966a) we may choose, for instance, the square root of the (relative) enstrophy

as the norm, and take

$$
\begin{gathered}
\|\psi\|_{-} \equiv\left\{\iint_{D}\left(\nabla^{2} \psi\right)^{2} \mathrm{~d} x \mathrm{~d} y\right\}^{\frac{1}{2}} \geqslant 0 \\
\delta^{2}=\frac{\left(c k_{0}^{2}-1\right) \epsilon^{2}}{C k_{0}^{2}}
\end{gathered}
$$

which is positive by virtue of (6.22). Then, if $\left\|\psi_{0}\right\|_{-}<\delta$,

$$
\begin{aligned}
\|\psi\|_{-}^{2} & =\iint_{D}\left(\nabla^{2} \psi\right)^{2} \mathrm{~d} x \mathrm{~d} y=\frac{k_{0}^{2}}{c k_{0}^{2}-1} \iint_{D}\left(c\left(\nabla^{2} \psi\right)^{2}-\frac{1}{k_{0}^{2}}\left(\nabla^{2} \psi\right)^{2}\right) \mathrm{d} x \mathrm{~d} y \\
& \leqslant \frac{k_{0}^{2}}{c k_{0}^{2}-1} \iint_{D}\left(c\left(\nabla^{2} \psi\right)^{2}-|\nabla \psi|^{2}\right) \mathrm{d} x \mathrm{~d} y \leqslant \frac{k_{0}^{2}}{c k_{0}^{2}-1} \iint_{D}\left(C\left(\nabla^{2} \psi_{0}\right)^{2}-\left|\nabla \psi_{0}\right|^{2}\right) \mathrm{d} x \mathrm{~d} y \\
& \leqslant \frac{C k_{0}^{2}}{c k_{0}^{2}-1} \iint_{D}\left(\nabla^{2} \psi_{0}\right)^{2} \mathrm{~d} x \mathrm{~d} y=\frac{C k_{0}^{2}}{c k_{0}^{2}-1}\left\|\psi_{0}\right\|_{-}^{2}<\epsilon^{2}
\end{aligned}
$$

where (6.21) and (6.22) have been used to yield the first inequality in the middle line, and (6.15) to yield the second. Again, (6.25) establishes stability in both the second (Liapunov) and the third sense. Note that, in contrast with the positive definite case, the relation (6.24) between $\epsilon$ and $\delta$ now depends explicitly on the domain geometry through $k_{0}$.

Stability in the second and third senses can also be proved with respect to the norms

$$
\left\{\iint_{D}\left\{c\left(\nabla^{2} \psi\right)^{2}-|\nabla \psi|^{2}\right\} \mathrm{d} x \mathrm{~d} y\right\}^{\frac{1}{2}}, \quad\left\{\iint_{D}\left\{C\left(\nabla^{2} \psi\right)^{2}-|\nabla \psi|^{2}\right\} \mathrm{d} x \mathrm{~d} y\right\}^{\frac{1}{2}},
$$

the relation between $\epsilon$ and $\delta$ then being sharpened to

$$
\delta^{2}=\frac{\left(c k_{0}^{2}-1\right) \epsilon^{2}}{C k_{0}^{2}-1}
$$

in both cases. The proof is very similar and again makes use of $(6.18)$ or $(6.21)$. These versions of the stability theorem are significantly more powerful than $(6.25)$ in cases where $C k_{0}^{2}-1$ is numerically small.

In the special case of a parallel or axisymmetric basic flow, the disturbance problem is Galilean-invariant; thus one may add a constant flow $\left(U_{0}, 0\right)$ of arbitrary strength to the basic flow without affecting the latter's stability properties. If $Q(y)$ is monotonic, then Arnol'd's first theorem is valid in the limit $U_{0} \operatorname{sgn} Q_{y} \rightarrow-\infty$, $\Psi^{\prime}(Q) \rightarrow \infty$, and the norm \|\|$_{+}$in (6.13) goes to \|\|$_{-}$as defined in (6.23). In this limit, $c$ and $C$ can be taken to $\infty$ in such a way that the ratio $C / c \rightarrow\left|Q_{y}\right|_{\max } /\left|Q_{y}\right|_{\min }$ in (6.13), giving the rigorous bound

$$
\iint q^{2}(x, y, t) \mathrm{d} x \mathrm{~d} y \leqslant \frac{\left|Q_{y}\right|_{\max }}{\left|Q_{y}\right|_{\min }} \iint q^{2}(x, y, 0) \mathrm{d} x \mathrm{~d} y
$$

valid for monotonic $Q(y)$. This is a nonlinear extension of Rayleigh's 'inflection-point ' stability theorem, for disturbances of arbitrary magnitude. It may equally well be derived directly using the generalized Eliassen-Palm or 'impulse-Casimir' invariant $J$ (cf. $\$ 7$ ), using a priori estimates in the same way as here.

We end this section with four examples. First, a simple and well-known example of stabilization by boundary constraints is parallel flow in a channel $-\Delta Y / 2<y<\Delta Y / 2$, with profiles

$$
U=(\sin y, 0), \quad Q=-\cos y .
$$


We have $k_{0}=(\pi / \Delta Y)$ and can take $c=C=1$, so that the 'sandwich' $(6.14)$ is infinitely thin. Arnol'd's second theorem implies that this flow will be nonlinearly stable to inviscid disturbances of any amplitude, despite the presence of an inflection point in the velocity profile, whenever the channel is narrow enough that

$$
\Delta Y<\pi
$$

(cf. Arnol'd 1965, example 2, figure 2). It will be noticed that in this particular case (4.1) is exact. $A$ is constant, so that $(4.3 a)$ is an exact quadratic invariant, and no information need be thrown away in order to express stability in terms of a norm: in fact both expressions in (6.26) are just $(-\mathscr{A})^{\frac{1}{2}}$ and are exact constants of the motion, unlike the enstrophy norm (6.23). The latter can grow, to an extent limited only by the looser bound (6.24). Note that $\epsilon / \delta$ from $(6.27)$ is unity whereas from $(6.24)$ it may be numerically large.

A second example of stabilization by boundary constraints is provided by the 'orographic' or self-tuning resonant instability, for instance in a channel with slightly wavy walls. The existence of the instability (when $\Psi^{\prime}(Q)<0$, and for certain values of the channel width) can be demonstrated analytically following Plumb (1981). The analysis (omitted here) verifies what is already evident from Arnol'd's second theorem, namely that this instability is also suppressed if, for given basic-flow profiles of both velocity and potential vorticity, the channel walls are sufficiently close together. The analysis also makes clear the physical reason for the suppression of the instability (Plumb 1981), namely that for given $\Psi$ and $Q$ the upstream phase speed of a Rossby wave diminishes with the channel width, so that if the channel is too narrow then the system will be too far from any stationary Rossby-wave resonance for the self-tuning mechanism to operate.

In fact the first example, stabilization of shear instabilities in a non-wavy channel, can also be shown to be related to the notion of upstream Rossby-wave propagation in a somewhat similar way (Lighthill 1963, p. 93; Bretherton 1966b; Hoskins et al. $1985, \S 6 b)$. The main difference is that the shear instability involves two or more counterpropagating Rossby waves, each propagating upstream so as to be able to be brought to rest by the local basic flow and to remain stationary relative to the other. Since $\Psi^{\prime}(Q)=-U / Q_{y}$ in this case, $\Psi^{\prime}(Q)<0$ implies that the propagation is upstream in each half of the channel, towards $-x$ when $y>0$ and towards $+x$ when $y<0$. The boundary constraint can reduce the intrinsic phase speed of this upstream propagation, destroying the ability of the two Rossby waves to keep in step and hence suppressing the instability.

A third example is that of a (wavy) basic flow having a single lengthscale $\kappa_{0}^{-1}$, for instance

$$
\Psi=\sum_{|\kappa|=\kappa_{0}} a(\kappa) \exp (\mathrm{i} \kappa \cdot x)
$$

where the summation is over an arbitrary number of wavenumbers $\kappa$ with the same magnitude but different directions, and where $f$ in (2.2) is constant. Arnol'd's second theorem implies stability if the disturbance $\psi$ is constrained to be of smaller scale than the basic flow, in the sense that

$$
\kappa_{\mathrm{m}}(\psi)>\kappa_{0} \text { for all } \psi,
$$

where $\kappa_{\mathrm{m}}$ is defined, as before, by (6.17). The Poincaré inequality (6.18) shows that the condition $(6.32)$ will be satisfied whenever

$$
k_{0}>\kappa_{0}
$$


which can be forced to hold true by introducing (wavy) boundaries which lie along two streamlines of (6.31) and which are sufficiently close to each other. For the basic flow (6.31) (of which (6.29) is a special case), the 'sandwich' (6.14) can again be taken infinitely thin, with

$$
c=C=1 / \kappa_{0}^{2} \text {. }
$$

All the foregoing statements are true also in the beta-plane case $(f=\beta y+$ constant $)$, provided that a constant zonal flow $U=\left(\beta / \kappa_{0}^{2}, 0\right)$ is added to the basic flow (6.31). Once again, no information need be thrown away in order to express stability in terms of a norm, and both norms in (6.26) are exact constants of the motion.

A fourth example is the exact Kelvin cat's-eye solution of Stuart (1967). As recently pointed out by Holm et al. (1985), Arnol'd's second theorem proves that this, too, is stable - provided that it is constrained by wavy boundaries sufficiently close to the cat's eyes. The basic flow is like that of figure 2, except that it is unifunctional with $f=$ constant and $\Psi^{\prime}(Q)<0$, so that the 'Loch Ness monster' of figure $2(c)$ collapses to a single (logarithmic) curve extending from bottom right to top left of the $(Q, \Psi)$-domain.

The scale restriction implied by the stability condition (6.33), equivalently (6.22) in the case of (6.31), suggests a connection with Fjørtoft's (1953) celebrated 'anti-cascade' theorem. The theorem (e.g. Charney 1973, p. 296; Merilees \& Warn 1975) implies that in nonlinear interactions involving different scales of a twodimensional, inviscid flow, the total kinetic energy and enstrophy (which are separately conserved) cannot be transferred either to larger scales exclusively, or to smaller scales exclusively. Indeed Fjørtoft's paper goes on to deduce that a stream function $\Psi$ proportional to the gravest spherical harmonic represents a nonlinearly stable flow on a sphere, in a certain sense (although his definition of disturbance amplitude is not, strictly speaking, a norm for the disturbance). But it should be cautioned that the connection between stability and the anti-cascade theorem is not so simple in general, because in most cases where $(6.33)$ holds the basic flow and the disturbance are not orthogonal to each other in terms of either energy or enstrophy, so that Fjørtoft's arguments about energy and enstrophy transfers in infinite or spherical domains cannot be used directly. Rather, as already emphasized, the simplest results are obtained from conservable disturbance properties like $A$. Another such disturbance property is the 'Eliassen-Palm' or 'impulse-Casimir' invariant $J$ defined in (7.11) below, which leads to the generalized Rayleigh theorem (6.28). The usefulness of conservable disturbance properties has recently been emphasized by Held (1985) in a discussion of stable, linear, normal-mode disturbances to shear flows.

The mathematical elegance of Arnol'd's results should not be allowed to distract attention from three further points which may be significant in applications. The first is that there are clearly cases, of which figure 1 is an example, especially if $\epsilon$ is small, which are probably stable but not provably so by Arnol'd's method. The second is that nonlinear stability, in any of the senses discussed, does not necessarily provide a sharp constraint on quantities which may be of interest like the disturbance energy

$$
\hat{\mathscr{E}} \equiv \iint_{D} \hat{E} \mathrm{~d} x \mathrm{~d} y .
$$

For instance it has been known since the work of Kelvin (Thomson 1887) and Orr (1907) on 'sheared disturbances' that initial conditions can be chosen such that $\hat{\mathscr{E}}$ 


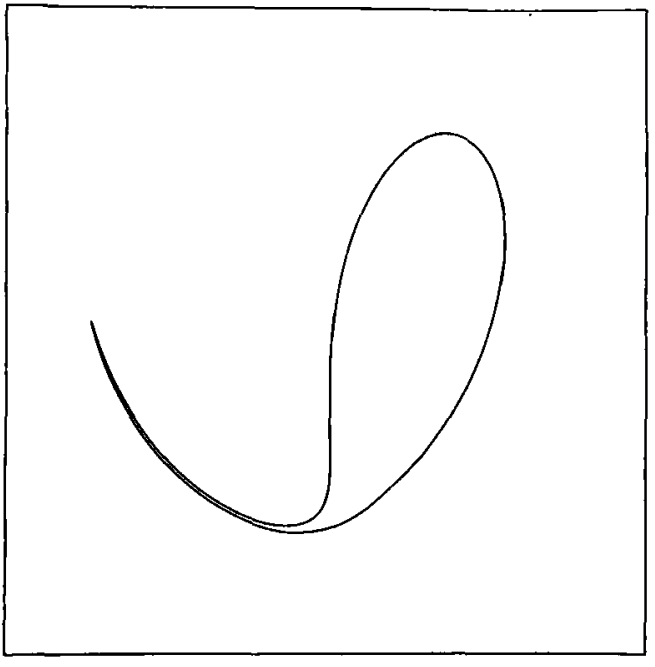

(a)

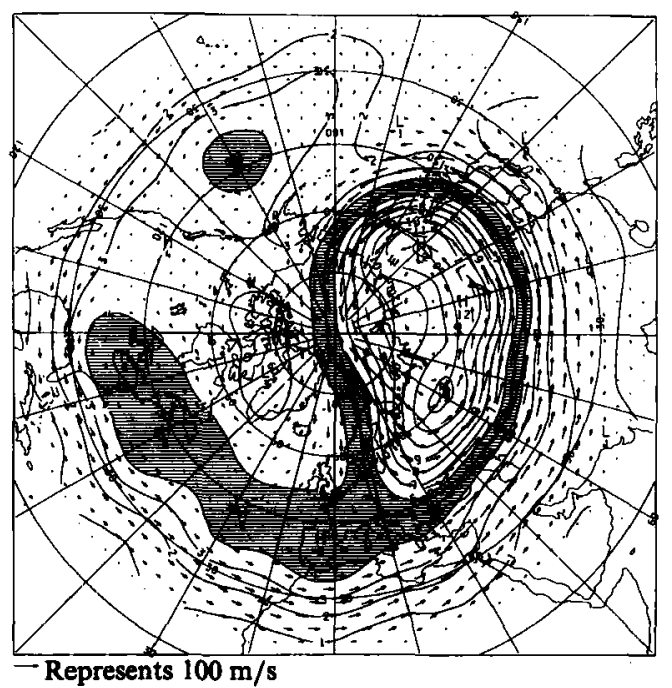

(b)

Figure 4. Two examples of breaking Rossby waves, $(a)$ on the free boundary of a vortex patch, from a numerical simulation by Dritschel (1986), and $(b)$ on the stratospheric polar-night vortex at an altitude of about $30 \mathrm{~km}$, from Clough et al. (1985). In $(b)$ the quantity plotted is the Rossby-Ertel potential vorticity on the $850 \mathrm{~K}$ isentropic surface. The small arrows show the associated velocity field. In both cases the undisturbed, axisymmetric basic flows are nonlinearly stable; for case (a) see Wan \& Pulvirenti (1985).

grows as large as we please for initial values (of $\widehat{\mathscr{E}}$ ) as small as we please.† Since $\mathscr{E}^{2}$ itself qualifies as a norm, this illustrates the well known fact that a given flow may be Liapunov stable with respect to one norm, but not with respect to another.

The third point is that stability, in any of the foregoing senses, does not rule out the possibility that disturbances may bring about significant irreversible changes in dynamical systems with an infinity of degrees of freedom, such as the fluid systems under consideration here. For instance, the breaking of Rossby waves propagating on a stable basic flow having a monotonic (potential) vorticity gradient will generally rearrange vorticity or potential vorticity irreversibly (e.g. Deem \& Zabusky 1978; Stewartson 1978; Warn \& Warn 1978; McIntyre \& Palmer 1984, 1985; Haynes 1985, 1987; Killworth \& McIntyre 1985; Juckes \& McIntyre 1987). A simple case is illustrated in figure 4(a) (from Dritschel 1986), showing an early stage in the process. Fundamentally similar phenomena have recently been recognized as being important in large-scale, nonlinearly stable stratospheric flows, both for dynamical and for tracer-transport purposes (McIntyre \& Palmer 1983, 1984; Grose 1984; Al-Ajmi, Harwood \& Miles 1985; Clough, Grahame \& O’Neill 1985; Leovy et al. 1985; Butchart $\&$ Remsberg 1986; Dunkerton \& Delisi 1986). An example from the real stratosphere, derived from modern satellite data, is shown in figure $4(b)$.

$\dagger$ Further illustrations in the case of constant basic shear have recently been given by, e.g., Farrell (1982), Boyd (1983), and Shepherd (1985); the essential phenomenon is the 'opening of a Venetian blind ' when a short-wave vorticity pattern is initially tilted against the shear of the basic flow. 


\section{Symmetries, conservation relations and Hamiltonian structure}

Finally, we show how the conservation relation (3.7), together with the generalized Eliassen-Palm and exact available-potential-energy theorems mentioned in $\S 1$, are related to the symmetries of the problem and to the concepts of Hamiltonian structure and Casimir invariant. This is the basic insight which makes it clear how to construct conservation relations of the type under discussion in other, more complicated problems.

As noted in $\S 3$, the difference energy $\Delta E$ defined by (3.9) or by

$$
\Delta E=\nabla \Psi \cdot \nabla \psi+\frac{1}{2}|\nabla \psi|^{2}=\nabla \Psi \cdot \nabla \psi+\hat{E}
$$

is conserved, and vanishes for zero disturbance. The quantity

$$
H=-\Psi q-\frac{1}{2} \psi q
$$

is yet another such conserved density, since it is evident from the relation $q=\nabla^{2} \psi$ that

$$
\Delta E-H=\nabla \cdot\left\{\left(\Psi+\frac{1}{2} \psi\right) \nabla \psi\right\} .
$$

Furthermore, there are many such conserved densities which differ from each other in a less trivial sense, i.e. not simply by a divergence. This is because we can add to any conserved density an expression of the form

$$
C(Q+q)-C(Q),
$$

where $C($.$) is an arbitrary function, since (2.1)$ implies that $\mathrm{D} C(Q+q) / \mathrm{D} t=0$, or equivalently

$$
\frac{\partial}{\partial t} C(Q+q)+\nabla \cdot\{(U+u) C(Q+q)\}=0
$$

if we use the relation $\boldsymbol{\nabla} \cdot(\boldsymbol{U}+\boldsymbol{u})=\mathbf{0}$. That is, the arbitrary function $C(Q+q)$ is itself a conserved density, with flux $(U+u) C(Q+q)$. Since $(\Psi, Q)$ is by definition a solution of $(2.1), C(Q)$ is also a conserved density, with flux $U C(Q)$.

As is well known, the conservation of total energy, and therefore of the difference energies $\Delta E$ and $H$, are related to the time invariance of the total-flow problem. By contrast, $C(Q+q)$ is an example of what is known in theoretical physics as (the density of) a Casimir invariant, or Casimir for short, as we shall see below. Such an invariant has the characteristic property of being conserved whether or not the given formulation of the problem, in this case the Eulerian description of vortex dynamics, possesses any symmetry whatever. A symmetry is, in fact, involved, but one which is 'invisible' in the formulation because certain generalized coordinates are ignorable, and have been ignored in arriving at the formulation. In the present case the ignored generalized coordinates are Lagrangian particle positions (or the equivalent information contained in a Clebsch-potential description, e.g. Salmon 1982, 1988).

It follows immediately from the preceding statements that, for example,

$$
H+C(Q+q)-C(Q)
$$

is a conserved density for any function $C($.) whatever. Following Holm et al. (1985), we might call the expression (7.5), or equally its counterpart with $H$ replaced by $\Delta E$, (the density of) an 'energy-Casimir invariant'. Now there is just one choice of the arbitrary function $C($.) (unique, at least, up to an additive constant) which makes the expression (7.5) into a second-order wave property (cf. Arnol'd 1965). That choice is

$$
C(\eta)=\int^{\eta} \Psi(\tilde{\eta}) \mathrm{d} \tilde{\eta} ;
$$


its uniqueness will be proved shortly. Not only does (7.5) then become a second-order wave property, but it is then nothing other than the density $A$ of Arnol'd's invariant defined in (3.8a), apart from the divergence of a vector wave property. Explicitly, substitution of (7.6) into (7.5) yields

$$
H+C(Q+q)-C(Q)=A-\nabla \cdot\left\{\frac{1}{2} \psi \nabla \psi\right\}
$$

as the reader can easily verify upon referring back to (3.2) and (3.8a). Note that the second term of (3.2) comes directly from the first term on the right of (7.2), that the first term on the right of $(3.2)$ is just $C(Q+q)-C(Q)$, and that the relation $q=\nabla^{2} \psi$ has again been used. As already emphasized, $\Psi(Q)$ and therefore (7.6) need not be single-valued, with the consequences noted in $\$ 5$. The possibility of choosing $C($.$) in the manner just described depends on the time symmetry of the basic state,$ since in order to be equal to the left-hand side, the right-hand side of (7.6) must be a function of $\eta$ alone.

Andrews (1983) remarks on the relation between the small-amplitude approximation to $A,(4.3 a)$, and the concept of pseudoenergy or, as some authors prefer to call it, quasi-energy. A pseudoenergy or quasi-energy may be generally defined to be a conservable second-order wave property, in the sense defined in $\$ 3$, whose conservation is linked to time symmetry of some mean or basic reference flow. $A$ is evidently a case in point. (It is to be sharply distinguished from energy, total or difference, whose conservation is linked to time symmetry of the total-flow problem and is indifferent to whether or not the basic flow is steady.) It is known that such steady-flow-related wave properties arise automatically, and in a very general way, in formulations where the disturbance is described by Lagrangian particle displacements about a reference state (e.g. Bretherton \& Garrett 1968; Andrews \& McIntyre $1978 b$; Ripa 1981). The foregoing shows how they can also arise within the Eulerian description, subject to certain conditions, the main requirement being that Casimir invariants are available having enough arbitrariness to allow choices like (7.6) to be made. Holm et al. (1985) and Abarbanel et al. $(1984,1986)$ give several other examples in the course of their interesting work on stability. The concepts of 'stability' and 'wave property' are related by the fact that a priori estimates like (6.10) evidently cannot be constructed unless the conserved functional $\mathscr{A}$ behaves quadratically for small disturbance amplitude.

It is arguable that the Lagrangian description is still involved in the construction of quantities like $A$, albeit implicitly. From the present example, together with the examples given in the works just cited, we may surmise that the requirement of sufficient arbitrariness in the available Casimirs is equivalent to requiring that the basic state have non-vanishing gradients of materially conserved quantities (such as specific entropy or potential vorticity) such that the disturbance fields carry the needed Lagrangian information about disturbance particle displacements (cf. Andrews \& McIntyre 1978a, appendix C). This for instance seems to be the essential clue to understanding an otherwise mysterious limiting process invoked by Abarbanel et al. $(1984,1986)$ in their analysis of stratified shear instability, in which a small spanwise isentropic gradient of potential vorticity is introduced into what at first sight seems to be a spanwise-independent, two-dimensional problem. A case where insufficient Casimirs exist to obtain a conserved, second-order, quasi-Eulerian wave property is that of a three-dimensional, incompressible homogeneous fluid (Holm et al. 1985).

This way of constructing conservable wave properties, exemplified by (7.5) and (7.6), need not be restricted to the case of time symmetry alone. For instance, if 
the total-flow problem is also translationally symmetric in the $x$-direction, then the density of Kelvin's impulse $(Q+q) y$ is conserved (e.g. Lamb 1932, $\S 152$; Batchelor 1967, p. 529; Benjamin 1984). Defining the disturbance contribution to the impulse density as

$$
I=q y,
$$

we can construct conserved 'impulse-Casimir' densities

$$
I+C(Q+q)-C(Q)
$$

analogous to (7.5). Once again, this expression can be made into a second-order wave property by just one choice, up to an additive constant, of the function $C($.), namely

$$
C(\eta)=-\int^{\eta} y_{0}(\tilde{\eta}) \mathrm{d} \tilde{\eta}
$$

where $y_{0}($.$) represents the functional dependence of the coordinate y$ upon $Q$ in an $x$-invariant basic state. As before (ef. (7.6) ff.), the symmetry property of the basic flow is a necessary condition for this choice to be possible, and therefore for the existence of a second-order disturbance invariant; and as before $y_{0}(Q)$ may be multivalued. The resulting conservable wave property, $I+C(Q+q)-C(Q)=J$, say, may be written as

$$
J(Q, q) \equiv-\int_{0}^{q}\left(y_{0}(Q+\tilde{q})-y_{0}(Q)\right) \mathrm{d} \tilde{q}
$$

which apart from sign convention is just the density of ' $\mathrm{EP}$ wave activity' appearing in the exact generalized Eliassen-Palm theorem noted by Killworth \& McIntyre (1985), and used by them to place bounds on Rossby-wave critical-layer absorption. The foregoing shows that the 'EP wave activity' $-J(Q, q) \approx \frac{1}{2}(\mathrm{~d} Q / \mathrm{d} y)^{-1} q^{2}+O\left(q^{3}\right)$ may be regarded as (the negative of) an Eulerian pseudomomentum, or quasimomentum (cf. Held 1985), as well as directly exhibiting its relation to Kelvin's impulse. In the same way it can be shown that the 'available potential energy' of an incompressible, stratified fluid, for which an exact formula of the type (3.2) or (7.11) was found by Holliday \& MeIntyre (1981, equation (2.15)), is the potentialenergy-associated contribution to a pseudoenergy or quasi-energy for which the time-invariant basic state is taken to be a motionless reference state in hydrostatic equilibrium. The relevant Casimir density takes the form of an arbitrary function of the fluid mass density, which is materially conserved. $\Psi$ in (3.2) then represents the basic gravitational potential and $Q+q$ the mass density; both can vary threcdimensionally. The corresponding compressible formula was found by Andrews (1981), in which case the materially conserved quantity is potential density or potential temperature, and the results are special cases of more general energyCasimir formulae found by Holm et al. (1985) and Abarbanel et al. (1986) in their studies of the stability shear flows. We note that the fluxes associated with these energy-Casimir and impulse-Casimir densities, e.g. the flux (3.8b), can be dircctly constructed by adding the appropriate difference energy or impulse flux to the difference Casimir flux $(U+u) C(Q+q)-U C(Q)$; details are omitted for brevity.

It remains to prove the uniqueness of (7.6) and (7.10) and to explain how the link between symmetries and conservation relations can be simply expressed in Hamiltonian form within the Eulerian description and how it follows, in particular, that $C(Q+q)$ does represent the density of a Casimir invariant, as that term is understood in theoretical physics.

To prove the uniqueness of (7.6) and (7.10) (up to an additive constant) we must prove that no other choices of the arbitrary function $C($.$) would make (7.5) and (7.9)$ 
into second-order wave properties. This will show in particular that if $C($.$) is$ multivalued then the multivaluedness, together with its consequences noted in $\$ 5$, is inescapable. We continue to regard $\Psi$ and $Q$ as fixed functions of $(x, y)$, and define

$$
\begin{gathered}
\mathscr{C}(q)=\iint_{D}\{C(Q+q)-C(Q)\} \mathrm{d} x \mathrm{~d} y, \\
\mathscr{I}(q)=\iint_{D} y q \mathrm{~d} x \mathrm{~d} y \\
\mathscr{H}(q)=\iint_{D} H \mathrm{~d} x \mathrm{~d} y,
\end{gathered}
$$

regarding these expressions as functionals of $q($.$) . In the last expression, we define$ the dependence upon the function $q($.$) to be that implied by rewriting (7.2) as$

$$
H=-\Psi q-\frac{1}{2} q \nabla^{-2} q,
$$

with the understanding that the boundary conditions (6.4) and (6.6) are used to make the inverse Laplacian unambiguous (up to an additive constant), and that $q$ satisfies (6.7). These definitions will also be used in connection with the discussion of Hamiltonian structure. Now consider the functionals

$$
\mathscr{H}+\mathscr{C}, \mathscr{I}+\mathscr{C} .
$$

If the corresponding densities $H+C(Q+q)-C(Q)$ and $I+C(Q+q)-C(Q)$ are to be second-order wave properties then so must the functionals $\mathscr{H}+\mathscr{C}$ and $\mathscr{I}+\mathscr{C}$ just defined. But then those functionals must be $O\left(q^{2}\right)$ for small but otherwise arbitrary $q$. This is possible only if their functional derivatives with respect to $q$ vanish when $q$ is identically zero (cf. Arnol'd 1965):

$$
\left.\frac{\delta(\mathscr{H}+\mathscr{C})}{\delta q}\right|_{q=0}=0,\left.\quad \frac{\delta(\mathscr{H}+\mathscr{C})}{\delta q}\right|_{q=0}=0 .
$$

Note incidentally that the use of functional derivatives, rather than ordinary partial derivatives, is a necessary device here: the presence of the non-local operator $\nabla^{-2}$ in (7.15) makes it meaningless to speak of a local derivative ' $\partial H / \partial q$ '. A sufficiently general definition of the operator $\delta / \delta q$ is given in Appendix C; see (C 1) and (C 12). Inspection of (7.12)-(7.15) shows that

and

$$
\frac{\delta \mathscr{C}}{\delta q}=C^{\prime}(Q+q), \quad \frac{\delta \mathscr{I}}{\delta q}=y,
$$

$$
\frac{\delta \mathscr{H}}{\delta q}=-\Psi+O(q) .
$$

Substituting these results into $(7.17 a, b)$ we get

$$
-\Psi+C^{\prime}(Q)=0, \quad y+C^{\prime}(Q)=0,
$$

which imply (7.6) and (7.10) respectively.

Of course $(7.17 a, b)$ are only necessary conditions for the corresponding densities to be wave properties; e.g. (7.17a) does not guarantee that the density (7.5) is locally second order. A divergence may have to be removed in general.

We now show that the disturbance problem defined by $(2.7),(6.4)$ and $(6.6)$ is Hamiltonian, with the set $\Pi$ of Eulerian disturbance (potential) vorticities $q(x, y, t)$ at a given time $t$ playing the role of the phase or state space. $\Pi$ is evidently a 'reduced' 
phase space, in that a large amount of Lagrangian information about particle positions is ignored; such reduction of the phase space is generally possible in the presence of symmetries (e.g. Abraham \& Marsden 1978, p. 298; Arnol'd 1978, appendix 5), in this case the particle-label symmetry. The present proof follows ideas that are freely available in the recent literature (e.g. Arnol'd 1969; Kuznetsov \& Mikhailov 1980; Morrison 1982; Marsden \& Weinstein 1983), although not all the details for the case of two-dimensional vortex dynamics seem to have been put on record before. The proof uses the appropriate non-canonical Poisson bracket and is presumed to be essentially equivalent (Olver 1983,1984$)$ to the less direct and more technically difficult proof given previously by Olver $(1980,1982)$ in terms of the corresponding symplectic differential two-form. The fact that the Hamiltonian structure carries over to the disturbance problem has previously been pointed out and exploited by Benjamin $(1984, \S 5.3)$. The present proof has the advantage of making the role of the boundary conditions explicit, particularly (6.4). An independent, and more general, investigation of the boundary conditions has recently been presented by Lewis et al. (1986).

To demonstrate Hamiltonian structure it is sufficient to find expressions for a Hamiltonian functional and Poisson bracket equivalent to (2.7), and to demonstrate that they have the requisite properties. The functions $q($.$) do not comprise a canonical$ representation of the phase space, and so the Poisson bracket (equivalently, the 'co-symplectic two-vector') will not be in classical, canonical form. Like the Dirac quantum-mechanical bracket it will be a generalized Poisson bracket, recognizable, as will be shown, by its abstract-algebraic properties.

The Hamiltonian functional may be taken as the difference energy $\iint \Delta E \mathrm{~d} x \mathrm{~d} y$ expressed as a functional of $q$. Under the boundary conditions (6.4) and (6.6), and the restriction (6.7), this is just $\mathscr{H}(q) .+$ We shall need to know its functional derivative when $q$ is not zero, extending (7.19). This follows from (7.15), (7.19) and the relation

$$
\frac{\delta}{\delta q} \iint_{D} q \nabla^{-2} q \mathrm{~d} x \mathrm{~d} y=2 \nabla^{-2} q=2 \psi,
$$

which, as Benjamin (1984) points out, is proved most readily by using the symmetry of the operator $\nabla^{-2}$, viz.

$$
\iint_{D} q_{1} \nabla^{-2} q_{2} \mathrm{~d} x \mathrm{~d} y=\iint_{D} q_{2} \nabla^{-2} q_{1} \mathrm{~d} x \mathrm{~d} y,
$$

another consequence of (6.4) and (6.6), and differentiating $q_{1}$ and $q_{2}$ separately. Thus

$$
\frac{\delta \mathscr{H}}{\delta q}=-\Psi-\psi
$$

The generalized Poisson bracket [.,.] for the disturbance problem (2.7) will be defined in the first instance to operate on pairs of functionals $\mathscr{F}(q), \mathscr{G}(q)$ whose functional derivatives with respect to $q$ satisfy the same kinematical boundary condition as $\Psi$ and $\psi$, namely (6.4), i.e. $\partial / \partial s=0$ on $\partial D$ for any function $q($.$) . For$ convenience we call these 'admissible functionals'; they include $\mathscr{H}(q)$, by (7.23). The

$\dagger$ If the restrictions implied by (6.6) and (6.7) were lifted, the Hamiltonian $\iint \Delta E \mathrm{~d} x \mathrm{~d} y$ would be equal to $\mathscr{H}=\mathscr{H}+\Sigma \Psi_{n} \gamma_{n}+\frac{1}{2} \Sigma \psi_{n} \gamma_{n}$, where $\gamma_{n}$ is the circulation $\oint u \cdot \mathrm{d} s$ around the $n$th connected portion $\partial D_{n}$ of $\partial D$ (ds positive with the fluid on the left). The notion of functional derivative of $\mathscr{H}$ must then be generalized to mean the collection of $(N+1)$ entities $\{\delta \hat{\mathscr{H}} / \delta q$, $\left.\partial \mathscr{\mathscr { H }} / \partial \gamma_{1}, \ldots, \partial \hat{\mathscr{H}} / \partial \gamma_{N}\right\}$ (e.g. Marsden \& Weinstein 1983; Lewis et al. 1986), so that the general variation $\delta \hat{\mathscr{H}}=\iint(\hat{\delta} \hat{\mathscr{H}} / \delta q) \delta q \mathrm{~d} x \mathrm{~d} y+\Sigma_{n}\left(\partial \hat{\mathscr{H}} / \partial \gamma_{n}\right) \mathrm{d} \gamma_{n}$. 
kinematical boundary condition enters because the properties of the Poisson bracket express, inter alia, the fact that the kinematics of the system can be described in terms of Lie groups and Lie algebras; see, e.g., Arnol'd $(1978, \S 40)$ and Marsden \& Weinstein $(1983, \S 2)$. The admissibility condition is satisfied if and only if $\mathscr{F}(q)$ and $\mathscr{G}(q)$ are functionals of state in a certain sense (see Appendix C). The generalized Poisson bracket is defined (for such admissible functionals; cf. Lewis et al. 1986) as

$$
[\mathscr{F}, \mathscr{G}]=\iint_{D}(Q+q) \partial\left(\frac{\delta \mathscr{F}}{\delta q}, \frac{\delta \mathscr{G}}{\delta q}\right) \mathrm{d} x \mathrm{~d} y
$$

As before, $\partial(.,$.$) denotes the Jacobian with respect to x, y$. Note that, since

$$
\iint_{D} f \partial(g, h) \mathrm{d} x \mathrm{~d} y=\iint_{D} g \partial(h, f) \mathrm{d} x \mathrm{~d} y=\iint_{D} h \partial(f, g) \mathrm{d} x \mathrm{~d} y
$$

for any three functions $f, g, h$ two of which are constant on each connected portion of $\partial D$, we can make the corresponding cyclic permutations in (7.24) provided that $\mathscr{F}(q)$ and $\mathscr{G}(q)$ are both admissible. The expression (7.24) has the abstract-algebraic properties characteristic of all classical and quantum-mechanical Poisson brackets, viz. bilinearity together with

$$
\begin{gathered}
{[\mathscr{F}, \mathscr{G}]=-[\mathscr{G}, \mathscr{F}],} \\
{[\mathscr{F} \mathscr{G}, \mathscr{K}]=[\mathscr{F}, \mathscr{K}] \mathscr{G}+\mathscr{F}[\mathscr{G}, \mathscr{K}],} \\
{[[\mathscr{F}, \mathscr{G}], \mathscr{K}]+[[\mathscr{G}, \mathscr{K}], \mathscr{F}]+[[\mathscr{K}, \mathscr{F}], \mathscr{G}]=0,}
\end{gathered}
$$

i.e. anticommutativity, the derivation property (applying to both arguments by (7.26a)), and satisfaction of Jacobi's identity. Here $\mathscr{F}(q), \mathscr{G}(q)$ and $\mathscr{K}(q)$ are any three admissible functionals. It can also be shown that the bracket of any two admissible functionals is itself an admissible functional. All these properties except the admissibility of [.,.], and Jacobi's identity (7.26 $c$ ), are obvious from the definition (7.24). The latter two properties are proved in Appendix C, where an extension of the class of admissible functionals, needed below, is also discussed.

As Marsden \& Weinstein (1983) explain, the expression (7.24) and its properties, including $(7.26 c)$, are formally deducible from a basic result originally proven by Lie for finite-dimensional systems (although the question of admissibility requires some attention to the boundary conditions, as already implied). The expression (7.24) can also be deduced directly by transformation from the canonical representation of the dynamics using the Lagrangian description of fluid motion (Lewis et al. 1986), in a manner that is straightforward apart from the treatment of the incompressibility and boundary constraints (discussed further in McIntyre 1987). The explicit expression (7.24) has previously been presented by Kuznetsov \& Mikhailov (1980), Morrison (1982) and Marsden \& Weinstein (1983), among others, and the essential idea can be traced back to Arnol'd (1969). Our contribution is merely to set on record the proof of (7.26c) given in Appendix C, using direct methods not dependent on a knowledge of the abstract theory.

It remains to show that the Hamiltonian functional $\mathscr{H}$ and the Poisson bracket (7.24) are equivalent to the equation of motion (2.7), which in turn is equivalent to (2.1) with $P=Q+q$ and $\Phi=\Psi+\psi$, by (2.4). The result of multiplying (2.1) by $\delta \mathscr{G} / \delta q$, for arbitrary admissible $\mathscr{G}$, and integrating over $D$, is

$$
\frac{\mathrm{d} \mathscr{G}}{\mathrm{d} t}=[\mathscr{G}, \mathscr{H}]
$$


by (7.23), (7.24) and (7.25). Conversely, we may recover (2.1) and (2.7), at any point $\left(x_{0}, y_{0}\right)$ within $\partial D$, by taking

$$
\mathscr{G}=\iint_{D} q(x, y, t) \delta\left(x-x_{0}\right) \delta\left(y-y_{0}\right) \mathrm{d} x \mathrm{~d} y=q\left(x_{0}, y_{0}, t\right)
$$

in (7.27), where $\delta($.$) is the Dirac delta function. (On the right of (7.27) we again$ use (7.25), as well as (7.24), together with $\delta \mathscr{G} / \delta q=\delta\left(x-x_{0}\right) \delta\left(y-y_{0}\right)$, from (C 12).) Thus (7.27), which is manifestly in Hamiltonian form, is equivalent to (2.7). Together with Appendix $\mathrm{C}$ this completes the proof that the disturbance problem (2.7) is Hamiltonian, under the boundary conditions (6.4) and (6.6).

We can now exhibit the relation between symmetries and conservation relations in what is perhaps its neatest general form covering the cases of interest here. Suppose that the admissible functional $\mathscr{G}(q)$ in (7.27) is the domain integral of a conserved density, not depending explicitly on the time, whose net flux across the boundary vanishes. Then $\mathscr{G}(q)$ is a constant of the motion, so that (7.27) becomes

$$
[\mathscr{G}, \mathscr{H}]=0 \text {. }
$$

But this statement has a dual meaning, as is well known. It can equally well be read as saying that $\mathscr{G}$ generates an infinitesimal symmetry operation, i.e. an infinitesimal change of state $\delta_{\mathscr{g}} q$ under which $\mathscr{H}$ is invariant (e.g. Dirac 1958, p. 115; Goldstein 1980, $\$ 9-5$; Whittaker 1937, §144; Benjamin 1984, eq. (1.4)). Thus (7.29) neatly expresses the fact that symmetries and conservation relations are virtually the same thing. In the present problem, (7.24) and (7.25) show that the operator $[\mathscr{G},$.$] can be$ written, after multiplication by an arbitrarily small number $\epsilon$, as

where $\delta_{\mathscr{G}} q$ is defined by

$$
\epsilon[\mathscr{G}, .]=\iint_{D} \frac{\delta(.)}{\delta q} \delta_{\mathscr{G}} q \mathrm{~d} x \mathrm{~d} y
$$

$$
\delta_{\mathscr{G}} q \equiv \epsilon \partial\left(Q+q, \frac{\delta \mathscr{G}}{\delta q}\right)
$$

The last relation makes it clear that, in the present problem, the change of state $\delta_{\mathscr{G}} q$ generated by $\mathscr{G}$ is nothing but the infinitesimal virtual motion of fluid elements whose stream function is proportional to $\delta \mathscr{G} / \delta q$, keeping $Q+q$ constant on particles.

The cases $\mathscr{G}=\mathscr{H}$ and $\mathscr{G}=\mathscr{I}$ are of special interest here. We see from (7.23) and $(7.18 b)$ that the symmetry operations generated by $\mathscr{H}$ and by $\mathscr{I}$ have stream functions $\delta \mathscr{H} / \delta q$ and $\delta \mathscr{I} / \delta q$ equal to $\Psi+\psi$ and $y$ respectively, ignoring signs. The corresponding virtual motions are, respectively, translation in time and translation in the $x$-direction, as expected. Note that in order for $\mathscr{I}$ to qualify both as a constant of the motion and as an admissible functional of state (so that $\delta . \mathscr{I} / \delta q=$ constant on $\partial D$ ) the boundary geometry as well as the rest of the problem must be translationally invariant, that is to say the boundaries must be parallel to the $x$-axis, since $\delta \mathscr{I} / \delta q=y$. This is to be expected, since the pressure forces at any boundary not parallel to the $x$-axis can cause changes in the impulse $\mathscr{I}$.

Now a Casimir invariant may be generally defined to be a functional $\mathscr{C}$ which is always annihilated by the generalized Poisson bracket (e.g. Schiff 1968, p. 209):

$$
[\mathscr{F}, \mathscr{C}]=0 \text { for all admissible } \mathscr{F} .
$$

In quantum-mechanical language one says that $\mathscr{C}$ 'Poisson-commutes', or just 'commutes', with any (admissible) $\mathscr{F}$. The dual implications of (7.32) are, first, that $\mathscr{C}$ is a constant of the motion (since in particular we can take $\mathscr{F}=\mathscr{H}$ ), and, second, 
that the operation $[\mathscr{C},$.$] generates zero change in any functional of state. This means$ that it cannot represent any symmetry operation that is visible in the phase space in which we are viewing the system.

A closer look at how this works in our case proves instructive. In order for (7.32) to be true, it is necessary that the function $\delta_{\mathscr{G}} q$ in (7.30) should vanish identically when a Casimir $\mathscr{C}$ is substituted for $\mathscr{G}$. As can be seen at once from (7.31), this requires that $\delta \mathscr{C} / \delta q$ be a function of $Q+q$ alone. It follows, therefore, not only that the functional $\mathscr{C}(q)$ defined in (7.12) is indeed a Casimir according to the definition (7.32), but also that all Casimirs in the present problem must have the form (7.12) (additive constants such as the second term, $-\iint C(Q) \mathrm{d} x \mathrm{~d} y$, being immaterial here). This in turn enables us to see directly why the symmetry operation associated with any Casimir $\mathscr{C}$ is invisible in the Eulerian formulation, i.e. in the phase space $\Pi$. The stream function $\delta \mathscr{C} / \delta q$ of that symmetry operation, being a function of $(Q+q)$ alone, moves fluid particles along contours of constant $Q+q$, keeping $Q+q$ constant on each particle, and so does not change the vorticity field.

There is one remaining technicality. Many of the problems of present interest will have both $Q$ and $Q+q$ constant on the boundary $\partial D$, making $\mathscr{C}$ an admissible functional in the sense defined above. However, this is not always the case. For example, one might be interested in a rotating-fluid problem in which the basic state is one of rest relative to a beta plane, with $Q$ varying along $\partial D$. Then the stream function of the invisible symmetry operation generated by $\mathscr{C}$ violates the kinematical boundary condition (6.4).

To include such cases we must extend the space of admissible functionals to include functionals of the general form (7.12); that is to say the admissible functionals are now considered to be those whose functional derivatives either satisfy a boundary condition of zero tangential derivative on $\partial D$ corresponding to (6.4), or are functions of $Q+q$ alone as in (7.18a), or are the result of adding the two types (in accordance with the elementary rules for function spaces spanned by more than one category of function). It is straightforward to verify that the bracket (7.24) still has all the required properties in this extended function space; this, also, is done in Appendix C.

It can now be seen that the conservable wave properties

$$
\mathscr{A}=\iint_{D} A \mathrm{~d} x \mathrm{~d} y, \quad \mathscr{J}=\iint_{D} J \mathrm{~d} x \mathrm{~d} y
$$

generate, respectively, time and space translations (across the fixed background given by the basic flow), just as $\mathscr{H}$ and $\mathscr{I}$ do. This follows immediately from the corresponding statements for $\mathscr{H}$ and $\mathscr{I}$ together with the fact that $\mathscr{A}$ and $\mathscr{J}$ are special cases of (7.16), as we saw earlier. By (7.32), only the first term of each expression $\mathscr{A}=\mathscr{H}+\mathscr{C}$ and $\mathscr{J}=\mathscr{I}+\mathscr{C}$ in (7.16) contributes to the symmetry operators $[\mathscr{A},$.$] and [\mathscr{J},$.$] , and so the symmetry operations are the same as for \mathscr{H}$ and $\mathscr{I}$. Together with the fact that $\mathscr{A}$ and $\mathscr{J}$ are second-order wave properties, and the fact that adding $\mathscr{C}$ has introduced no explicit time-dependence into $\mathscr{A}$ and $\mathscr{J}$, this confirms their status as an Eulerian pseudoenergy or quasi-energy, and an Eulerian pseudomomentum or quasi-momentum respectively.

We are grateful to T. B. Benjamin who first drew our attention to the explicitly Hamiltonian structure of the Eulerian description of vortex dynamics, to J. Pedlosky for raising the issue of nonlinear stability in the third sense discussed in $\$ 6$ and to J. E. Marsden and his collaborators for showing us several of their interesting 
papers in advance of publication. We also thank D. G. Andrews, J. Gibbons, J. E. Marsden, R. T. Pierrehumbert, R. Salmon and T. Warn for helpful discussions and correspondence, and B. Haigh for translating Arnol'd (1969). T.G.S. is supported by the Natural Sciences and Engineering Research Council of Canada and by the UK Natural Environment Research Council.

\section{Appendix A. Barotropic flow on a rotating sphere}

For incompressible, two-dimensional flow on the surface of a rotating sphere, the potential vorticity equation $(2.1)$ takes the form

$$
\frac{\mathrm{D} P}{\mathrm{D} t} \equiv P_{t}+\frac{1}{a^{2} \cos \phi}\left(\Phi_{\lambda} P_{\phi}-\Phi_{\phi} P_{\lambda}\right)=0,
$$

with

$$
\left.\begin{array}{rl}
P & \equiv \nabla^{2} \Phi+f(\lambda, \phi) \\
& =\frac{1}{a^{2} \cos ^{2} \phi} \Phi_{\lambda \lambda}+\frac{1}{a^{2} \cos \phi}\left(\cos \phi \Phi_{\phi}\right)_{\phi}+f(\lambda, \phi),
\end{array}\right\}
$$

where $\lambda$ is longitude, $\phi$ latitude, $a$ the (constant) radius of the sphere, $\Phi$ the stream function and $f$ has the same significance as in (2.2). The manipulations of $\$ \S 2,3$ go through to yield the same formulae as before, $(3.7)$ and $(3.8 a-d)$, but with the appropriate interpretation of symbols such as $\nabla^{2}$ and $\nabla$ :

$$
\begin{gathered}
q=\nabla^{2} \psi=\frac{1}{a^{2} \cos ^{2} \phi} \psi_{\lambda \lambda}+\frac{1}{a^{2} \cos \phi}\left(\cos \phi \psi_{\phi}\right)_{\phi} \\
\nabla \psi=\frac{\psi_{\lambda}}{a \cos \phi} \lambda+\frac{\psi_{\phi}}{a} \hat{\phi},
\end{gathered}
$$

and similarly for the gradients of other scalars, where $\hat{\lambda}$ and $\hat{\phi}$ are the unit vectors in the longitudinal and latitudinal directions. 'Sphericity' terms arise in the spherical version of $(3.8 c)$ but cancel each other. Note that when calculating $D(\nabla \psi) / D t$ in $(3.8 c)$, care must be taken to use the correct curvilinear form of $[(U+u) \cdot \nabla] \nabla \psi$.

\section{Appendix B. Baroclinic, quasi-geostrophic flow}

Here we generalize the results of $\$ \S 2-4$ to the case of a three-dimensional, baroclinic, quasi-geostrophic fluid on a beta plane. The governing equation (2.1) still applies, but now does so at each vertical level $z$ (where $z$ is a log-pressure vertical coordinate), and $P$ is the quasi-geostrophic potential vorticity

$$
P \equiv \Phi_{x x}+\Phi_{y y}+\frac{1}{\rho}\left(\rho\left(\frac{f_{0}}{N}\right)^{2} \Phi_{z}\right)_{z}+f_{0}+\beta y ;
$$

here $\rho(z)$ is the reference-state density stratification and $N(z)$ the reference-state buoyancy frequency (see e.g. Pedlosky 1979, §6.5). Then the steady basic flow satisfies (2.4) at each $z$, and the functions $\Psi(Q)$ and $B(Q,$.$) introduced in (3.1) and$ (3.2) are functions also of $z$. Following manipulations which are closely analogous to those of $\$ \S 2,3$, we may immediately write the generalized form of the conservation theorem (3.7), namely

$$
\frac{\partial}{\partial t} A+\nabla_{3} \cdot F=0
$$


where

$$
A=\frac{1}{2} \rho\left|\nabla_{2} \psi\right|^{2}+\frac{1}{2} \rho\left(\frac{f_{0}}{N}\right)^{2} \psi_{z}^{2}+\rho B(Q, q ; z) \equiv \rho(\hat{E}+B),
$$

say, and where (cf. (3.8b))

$$
\begin{gathered}
F=\rho[B(Q, q ; z)-q \psi](U+u)-\rho \psi \frac{\partial}{\partial t}\left(\nabla_{2} \psi+\left(\frac{f_{0}}{N}\right)^{2} \psi_{z} \hat{z}\right)+\frac{1}{2} \rho \psi^{2} \hat{z} \times \nabla_{2} Q, \\
q \equiv \psi_{x x}+\psi_{y y}+\frac{1}{\rho}\left(\rho\left(\frac{f_{0}}{N}\right)^{2} \psi_{z}\right)_{z}
\end{gathered}
$$

or alternatively (cf. $(3.8 c))$

$$
\begin{aligned}
\boldsymbol{F}= & A(U+u)-\rho \psi \frac{\mathrm{D}}{\mathrm{D} t}\left(\nabla_{2} \psi+\left(\frac{f_{0}}{N}\right)^{2} \psi_{z} \hat{z}\right)+\frac{1}{2} \rho \psi^{2} \hat{z} \times \nabla_{2} Q+\frac{1}{2} \rho \partial(\psi, \Psi) \nabla_{2} \psi \\
& +\frac{1}{2} \rho \psi\left(\nabla_{2} \psi \cdot \nabla_{2} U-\nabla_{2} \Psi \cdot \nabla_{2} u\right)-\left\{\rho\left(\frac{f_{0}}{N}\right)^{2} \psi_{z}\right\}_{z} \psi(U+u)-\frac{1}{2} \rho\left(\frac{f_{0}}{N}\right)^{2} \psi_{z}^{2} U \\
& +\rho\left(\frac{f_{0}}{N}\right)^{2} \psi\left\{\psi_{z} \hat{z} \times \nabla_{2} \psi_{z}+(U+u) \cdot \nabla_{2} \psi_{z} z\right\}
\end{aligned}
$$

Here $\nabla_{3} \equiv\left(\partial_{x}, \partial_{y}, \partial_{z}\right), \nabla_{2} \equiv\left(\partial_{x}, \partial_{y}, 0\right)$ and $\hat{z}$ is the unit vertical vector. Note that in quasi-geostrophic theory, the advecting velocity field $(U+u)$ is purely horizontal. It may be verified that the forms (B $2 b, d$ ) of the conserved flux both possess the group-velocity property when the notion of group velocity applies. Equation (B 2d) is equal to $(\mathrm{B} 2 b)$ plus the identically non-divergent term $\hat{z} \times \nabla_{2}\left(\psi \hat{E}+\frac{1}{2} \psi U \cdot u\right)$.

In the special case of constant $N$, one may re-scale the vertical coordinate via $\zeta=N z / f_{0} ;$ then (B 1) holds with $\nabla_{3} \equiv\left(\partial_{x}, \partial_{y}, \partial_{\zeta}\right)$, and (B $\left.2 a, b\right)$ are simplified to

$$
\begin{gathered}
A=\frac{1}{2} \rho\left|\nabla_{3} \psi\right|^{2}+\rho B(Q, q ; \zeta), \\
F=\rho[B(Q, q ; \zeta)-q \psi](U+u)-\rho \psi \frac{\partial}{\partial t} \nabla_{3} \psi+\frac{1}{2} \rho \psi^{2} \hat{z} \times \nabla_{2} Q .
\end{gathered}
$$

To take the small-amplitude limit of (B $2 a, b)$ we follow the same procedures as in $\$ 4$. Additionally we restrict attention to a Boussinesq fluid, to facilitate direct comparison with Andrews (1983), whence $\rho$ can be taken constant and removed from (B 2), and $z$ becomes the height coordinate. The quasi-geostrophic analogue to $(4.3 a, b)$ is then

$$
\begin{gathered}
A \approx \frac{1}{2}\left|\nabla_{2} \psi\right|^{2}+\frac{1}{2}\left(\frac{f_{0}}{N}\right)^{2} \psi_{z}^{2}+\frac{q^{2}}{2 \Lambda}, \\
F \approx \frac{1}{2 \Lambda}(q-\Lambda \psi)^{2} U-\psi \frac{\partial}{\partial t}\left\{\nabla_{2} \psi+\left(\frac{f_{0}}{N}\right)^{2} \psi_{z} \hat{z}\right\},
\end{gathered}
$$

which is precisely Andrews' (1983) small-amplitude conservation theorem. The small-amplitude form of (B $2 d$ ), analogous to (4.3c), is

$$
\begin{aligned}
F-A U \approx & -\psi \frac{D_{\mathrm{b}}}{\mathrm{D} t}\left\{\nabla_{2} \psi+\left(\frac{f_{0}}{N}\right)^{2} \psi_{z} \hat{z}\right\}+\frac{1}{2} \psi^{2} \hat{z} \times \nabla_{2} Q+\frac{1}{2} \partial(\psi, \Psi) \nabla_{2} \psi \\
& +\frac{1}{2} \psi\left(\nabla_{2} \psi \cdot \nabla_{2} U-\nabla_{2} \Psi \cdot \nabla_{2} u\right)-\left[\left(\frac{f_{0}}{N}\right)^{2} \psi_{z}\right]_{z} \psi U-\frac{1}{2}\left(\frac{f_{0}}{N}\right)^{2} \psi_{z}^{2} U \\
& +\left(\frac{f_{0}}{N}\right)^{2} \psi U \cdot \nabla_{2} \psi_{z} \hat{z}
\end{aligned}
$$


Recently Plumb (1985b) has presented an alternative small-amplitude expression for the flux $(\mathrm{B} 3 b, c)$ (the flux being, of course, determinate only to within a non-divergent vector), which may be more useful in certain applications.

The derivation of a linear stability criterion for the case of positive $\Psi^{\prime}(Q)$, following the variational method of Arnol'd (1965), was performed for quasi-geostrophic flow with surface potential-temperature gradients by Blumen (1968) and by Dikiy \& Kurganskiy (1971), and further extended to include (smooth) topography by Charney \& Flierl (1981). Holm et al. (1985) have given the nonlinear treatment analogous to Arnol'd's $(1966 a)$ first stability theorem (i.e. positive $\left.\Psi^{\prime}(Q)\right)$ for the case of multilayer flow over topography, without surface potential-temperature gradients. In the present, continuous ease, it is formally a simple matter to include any surface potential-temperature gradients and topography within a $\delta$-function potential vorticity distribution just inside the boundaries, in the manner of Bretherton (1966a); this eliminates explicit boundary terms and makes the baroclinic problem essentially equivalent in analytical structure to the barotropic one. (This technique is reviewed in Hoskins et al. $(\mathbf{1 9 8 5}, \S 5 b)$.) However, since the nonlinear stability conditions for the continuous, positive definite case have not previously been noted, we shall give their explicit form here. We also note a somewhat restricted theorem for the negative definite case.

Returning to the non-Boussinesq form of the equations, the lower boundary condition is taken to be

$$
\frac{\mathrm{D}}{\mathrm{D} t}\left(\Theta+\theta+\lambda h_{\mathbf{B}}\right)=0 \quad \text { at } z=z_{1},
$$

where $\Theta(x, y, z)+\theta(x, y, z, t)$ is the deviation from a (prescribed) reference potentialtemperature distribution $\Theta_{\mathrm{S}}(z), h_{\mathrm{B}}(x, y)$ is the surface topographic height, assumed to be much smaller than any height scale of the flow, and $\lambda=\mathrm{d} \Theta_{\mathrm{s}} / \mathrm{d} z$. We write $\Theta+\theta$ to indicate the basic flow plus disturbance, as elsewhere and recall that in quasigeostrophic theory

$$
\Theta=\frac{\Theta_{\mathrm{s}} f_{0}}{g} \Psi_{z}, \quad \theta=\frac{\Theta_{\mathrm{s}} f_{0}}{g} \psi_{z} .
$$

An upper rigid boundary may exist, in which case

$$
\frac{\mathrm{D}}{\mathrm{D} t}(\Theta+\theta)=0 \quad \text { at } z=z_{2},
$$

or the domain may be semi-infinite, in which case an exponentially-growing disturbance is evanescent and in particular the vertical disturbance energy flux and the vertical flux of Arnol'd's invariant must vanish as $z \rightarrow \infty$, e.g.

$$
\lim _{z \rightarrow \infty} \iint \rho\left(\frac{f_{0}}{N}\right)^{2} \psi \psi_{z t} \mathrm{~d} x \mathrm{~d} y=0
$$

(see Charney \& Pedlosky 1963; Pedlosky 1979, §6.7; McIntyre \& Weissman 1978). If (B $4 c)$ can be assumed then all 'upper-boundary' effects vanish, so we give the treatment here assuming $(\mathrm{B} 4 a, b)$.

A steady 'basic' flow, with $\theta=q=0$, is characterized by $(\Psi, Q)$ satisfying (2.1) (or (2.4)) in the interior of the fluid, and by $(\Psi, \Theta)$ at the upper and lower boundaries satisfying the steady versions of (B $4 a, b)$. This allows the treatment of surface potential-temperature gradients and of smooth, shallow topography. And just as a 
functional relation exists between $\Psi$ and $Q$, namely $\Psi=\Psi(Q)$, for each $z$, so we have

$$
\begin{gathered}
\Psi=\Psi_{1}\left(\Theta+\lambda h_{\mathrm{B}}\right) \quad \text { at } z=z_{1}, \\
\Psi=\Psi_{2}(\Theta) \text { at } z=z_{2} .
\end{gathered}
$$

Consequently we may define functions $B_{1}\left(\Theta+\lambda h_{\mathrm{B}},.\right)$ at $z=z_{1}$ and $B_{2}(\Theta,$.$) at z=z_{2}$, just as $B(Q,$.$) is defined in the interior according to (3.2) (at each z$ ), only in this case the functional relations (B $5 a, b$ ) are used in place of (3.1), and the functional $\operatorname{argument}$ is $\theta$ rather than $q$. It is readily verified that, analogously to (3.6),

$$
\begin{gathered}
\frac{\mathrm{D}}{\mathrm{D} t} B_{1}\left(\Theta+\lambda h_{\mathrm{B}}, \theta\right)=-\theta \partial(\psi, \Psi) \quad \text { at } z=z_{1}, \\
\frac{\mathrm{D}}{\mathrm{D} t} B_{2}(\Theta, \theta)=-\theta \partial(\psi, \Psi) \quad \text { at } z=z_{2} .
\end{gathered}
$$

Then integrating (B 1) over the domain, using (6.4) and (6.6) to eliminate the horizontal boundary terms, and (B $4 a, b)$ and $(\mathrm{B} 6 a, b)$ to rewrite the vertical ones, it follows that

$$
\frac{\mathrm{d}}{\mathrm{d} t}\left\{\iiint A \mathrm{~d} x \mathrm{~d} y \mathrm{~d} z+\left.\iint \frac{\rho f_{0}}{\lambda} B_{1}\left(\Theta+\lambda h_{\mathrm{B}}, \theta\right) \mathrm{d} x \mathrm{~d} y\right|_{z=z_{1}}-\left.\iint \frac{\rho f_{0}}{\lambda} B_{2}(\Theta, \theta) \mathrm{d} x \mathrm{~d} y\right|_{z=z_{2}}\right\}=0 .
$$

As with $B(Q, q)$, the sign of $B_{1}$ or $B_{2}$ in the boundary terms is the same as the sign of $\Psi_{1}^{\prime}\left(\Theta+\lambda h_{\mathrm{B}}\right)$ or $\Psi_{2}^{\prime}(\Theta)$, whenever the latter are definite. Thus we may immediately state the extension of Arnol'd's first stability theorem to cover the present context of quasi-geostrophic flow: a basic flow $(\Psi, Q, \Theta)$ is stable if there exist constants $c, C$, $c_{1}, C_{1}$ and $c_{2}, C_{2}$ such that

$$
\begin{gathered}
0<c \leqslant \Psi^{\prime}(Q) \leqslant C<\infty \quad \forall z \in\left[z_{1}, z_{2}\right], \\
0<c_{1} \leqslant \Psi_{1}^{\prime}\left(\Theta+\lambda h_{\mathrm{B}}\right) \leqslant C_{1}<\infty \quad \text { at } z=z_{1},
\end{gathered}
$$

and

$$
0<c_{2} \leqslant-\Psi_{2}^{\prime}(\Theta) \leqslant C_{2}<\infty \quad \text { at } z=z_{2} \text {. }
$$

The stability which then follows is stability in the third, strongest sense discussed in $\$ 6$, and is with respect to the norm which is the natural quasi-geostrophic extension of (6.11), including boundary terms. Rather than (6.12), we have instead that

$$
\frac{\delta^{2}}{\epsilon^{2}}=\min \left\{\frac{c}{C}, \frac{c_{1}}{C_{1}}, \frac{c_{2}}{C_{2}}\right\}
$$

The extension of Arnol'd's second theorem in the general case is, by contrast, far from trivial. The difficulty is that the Poincaré inequality $(6.21)$, on which the proof of the second theorem depends in the two-dimensional case, fails to generalize straightforwardly in the presence of non-vanishing boundary terms. If, however, attention is restricted to the special case of homogeneous boundary conditions, namely

$$
\theta=0, \quad \text { i.e. } \psi_{z}=0, \quad \text { at } z=z_{1}, \quad z=z_{2}
$$

rather than (B 4a,b), then the generalization is straightforward. Then the boundary integrals of (B 7) vanish, and Arnol'd's second stability theorem takes the following form: a basic flow $(\Psi, Q)$ is stable if there exist constants $c$ and $C$ such that

$$
0<c \leqslant-\Psi^{\prime}(Q) \leqslant C<\infty \quad \forall z \in\left[z_{1}, z_{2}\right]
$$

and

$$
c k_{0}^{2}>1 \text {, }
$$


where $k_{0}$ is the positive number whose square is the lowest non-trivial eigenvalue of the problem

$$
\nabla_{2}^{2} \psi+\frac{1}{\rho}\left(\rho\left(\frac{f_{0}}{N}\right)^{2} \psi_{z}\right)_{z}=-k^{2} \psi
$$

in the interior, subject to $(6.4),(6.6)$ and (B 10). It is easily verified that the non-trivial eigenvalues of this problem are indeed positive. As in $\$ 6$, here (B $11 b$ ) ensures the negative definiteness of the functional

$$
\iiint \rho\left(\hat{E}-\frac{1}{2} c q^{2}\right) \mathrm{d} x \mathrm{~d} y \mathrm{~d} z
$$

which is what is needed to make the proof of stability go through. Using the obvious quasi-geostrophic extensions of the disturbance norms (6.23) or (6.26) (without boundary terms, of course), the $\epsilon-\delta$ relations (6.24) or (6.27) are again obtained. One can similarly extend the generalized Rayleigh theorem (6.28).

Some of the foregoing stability results have been noted independently by Swaters (1986). The Hamiltonian structure of the baroclinic quasi-geostrophic problem has been discussed by Holm (1986).

\section{Appendix C. Functionals of state, Casimir functionals and Jacobi's identity}

We write $(x, y)=x \in D$ for brevity, and suppress reference to the $t$-dependence in $q(x, t)$. First recall that if $\mathscr{F}\{q()$.$\} is any suitably well-behaved functional which maps$ real-valued functions $q(x)$ into real numbers, then $\delta \mathscr{F} / \delta q$, the functional derivative of $\mathscr{F}$, is defined to be that function of $x$ for which the change in $\mathscr{F}$ due to a small variation $\delta q$ in the function $q$ is given by

$$
\mathscr{F}(q+\delta q)-\mathscr{F}(q)=\iint_{D} \frac{\delta \mathscr{F}}{\delta q} \delta q(x) \mathrm{d}^{2} x+O\left(\delta q^{2}\right) .
$$

In the present problem the function $\delta \mathscr{F} / \delta q$ is defined only up to an additive constant, since $\iint \delta q \mathrm{~d}^{2} x=0$ under the restriction (6.7). As well as being a function of $x$ for any given $q(),. \delta \mathscr{F} / \delta q$ can also be considered to be a functional of $q($.$) for any given \boldsymbol{x}$. It will be convenient to indicate both dependences explicitly by writing

$$
\frac{\delta \mathscr{F}}{\delta q}=\frac{\delta \mathscr{F}\{q(\cdot)\}}{\delta q(x)} .
$$

We shall also need to make use of the second functional derivative, in the general sense involving two independent variations $\delta q_{1}(x), \delta q_{2}(x)$ :

$$
\begin{aligned}
& \mathscr{F}\left(q+\delta q_{1}+\delta q_{2}\right)-\mathscr{F}\left(q+\delta q_{1}\right)-\mathscr{F}\left(q+\delta q_{2}\right)+\mathscr{F}(q) \\
&=\iiint \int_{D \times D} \frac{\delta^{2} \mathscr{F}\{q(.)\}}{\delta q\left(x_{1}\right) \delta q\left(x_{2}\right)} \delta q_{1}\left(x_{1}\right) \delta q_{2}\left(x_{2}\right) \mathrm{d}^{2} x_{1} \mathrm{~d}^{2} x_{2}+O\left(\delta q^{3}\right)
\end{aligned}
$$

As the notation is meant to suggest, the second functional derivative appearing inside the integral is a function of two independently chosen points $x_{1}, x_{2} \in D$, and is also, of course, a functional of $q($.$) for fixed x_{1}$ and $x_{2}$. It can be regarded as the result of operating with $\delta / \delta q\left(x_{1}\right)$ upon the functional dependence, i.e. the $\{q()$.$\} dependence,$ of $\delta \mathscr{F}\{q().\} / \delta q\left(x_{2}\right)$, holding $x_{2}$ fixed. By the symmetry of the first line of (C 3$)$ we may 
equally well operate in the reverse order. Thus the second functional derivative has the symmetry property analogous to the symmetry of mixed partials in ordinary calculus:

$$
\frac{\delta^{2} \mathscr{F}\{q(.)\}}{\delta q\left(x_{1}\right) \delta q\left(x_{2}\right)}=\frac{\delta^{2} \mathscr{F}\{q(.)\}}{\delta q\left(x_{2}\right) \delta q\left(x_{1}\right)},
$$

again to within an additive constant in our case. This property will be needed in the proof of Jacobi's identity.

The concept of 'functional of state' used in $\$ 7$ is motivated and defined as follows. In classical dynamics (and in connection with the classical Poisson bracket in particular), the state of a system is considered to be specified when the positions and velocities of all the material particles comprising the system are specified. Thus a function or functional of state means a real-valued quantity that depends on the same information, i.e. depends only on the positions and velocities of the material particles. However, in the fluid system under consideration here, any two material particles are physically indistinguishable, in the sense that if they are interchanged, without altering the velocity field, then the state of the system is not observably different from what it was before. With this in mind, we define a functional of state in the present problem to mean a real-valued quantity which depends on the velocity field alone. $\dagger$

It was asserted in $\$ 7$ that $F(q)$ is a functional of state in the foregoing sense if, and only if, it is admissible in the sense defined below (7.23). That is, $\mathscr{F}(q)$ is a functional of state if, and only if,

$$
\frac{\partial}{\partial s}\left(\frac{\delta \mathscr{F}\{q(.)\}}{\delta q(x)}\right)=0 \quad \forall x \in \partial D, \quad \forall q(.) .
$$

Here $\partial / \partial s(s=$ arclength) operates on the $x$-dependence of $\delta \mathscr{F} / \delta q$. Equivalently, $\mathscr{F}$ is a functional of state if, and only if,

$$
\frac{\delta \mathscr{F}\{q(.)\}}{\delta q\left(x_{1}\right)}-\frac{\delta \mathscr{F}\{q(\cdot)\}}{\delta q\left(x_{2}\right)}=0
$$

for all functions $q($.$) and for any two points x_{1}, x_{2}$ on the same connected portion $\partial D_{n}$ of $\partial D$. (As mentioned in $\$ 7$, these conditions are not necessarily satisfied by the Casimir functionals $\mathscr{B}(q)$, which are dealt with separately below.)

The assertion may be verified as follows. To begin with, note that the expression on the left of (C 6), when multiplied by a small parameter $\epsilon$, is equal to the first-order change $\delta \mathscr{F}$ in when $\delta q$ is taken as

$$
\delta q=\epsilon\left\{\delta\left(x-x_{1}\right)-\delta\left(x-x_{2}\right)\right\}
$$

on the right of (C 1). Here $\delta$ denotes the two-dimensional Dirac delta function, and the expression is to be considered in the limit as $x_{1}, x_{2}$ approach positions on $\partial D_{n}$. The corresponding change $\delta u(x)$ in the velocity field has stream function

$$
\delta \psi=\epsilon \nabla^{-2}\left\{\delta\left(x-x_{1}\right)-\delta\left(x-x_{2}\right)\right\}
$$

As before, the boundary conditions (6.4) and (6.6) are used to make the linear operator $\nabla^{-2}$ uniquely defined up to an additive constant. Now consider the circulation $\delta \gamma_{n}$

$\dagger$ The same definition is implicit in Arnol'd's view of two-dimensional vortex dynamics, which can be expressed entirely in terms of the Eulerian velocity field, with no direct reference to Lagrangian particle labels (e.g. Holm et al. 1985, p. 17). 
of $\delta u$ around a closed contour enclosing $x_{1}, x_{2}$ and drawn arbitrarily close to $\partial D_{n}$. Stokes' theorem and the boundary conditions (6.4) and (6.6) imply that $\delta \gamma_{n}=0$. Therefore the velocity-field change $\delta u(x)$ is a potential flow with vanishing circulations around all the $\partial D_{n}$, and must therefore itself vanish identically. It follows that if $\mathscr{F}$ depends on the velocity field alone, then the change in $\mathscr{F}, \epsilon$ times (C 6), must likewise vanish. In other words if $\mathscr{F}$ is a functional of state in the foregoing sense, then (C 6) must indeed hold true, and so therefore must (C 5).

We must also demonstrate the converse, i.e. demonstrate that if $\mathscr{F}$ is not a functional of state then there must exist a function $q($.$) , and points x, x_{1}$ and $x_{2}$ lying somewhere on $\partial D$, such that (C 5) or equivalently (C 6) is violated. Now if $\mathscr{F}$ is not a functional of state, then there must exist (generalized) functions $q(x)$ and $\delta q(x)$ such that the corresponding $\delta \mathscr{F}$ defined by (C 1) does not vanish, whereas the change in the velocity field, $\delta u(x)$, induced by $\delta q(x)$, does vanish identically so that

$$
\nabla^{-2}\{\delta q(x)\} \equiv \text { constant. }
$$

Now (C 9) implies, first, that the $\delta q(x)$ in question must be concentrated wholly on the boundary $\partial D$, either as point vortices in the manner exemplified by (C 7) or as a vortex sheet, or both. For (C 9) would certainly be contradicted if $\delta q(x) \neq 0$ at any location away from the boundary $\partial D$. (Equivalently, a soap film or stretched elastic membrane cannot remain flat when a non-zero normal force is exerted on it anywhere away from the supporting boundaries.) Second, (C 9) implies that the vortex strength associated with $\delta q(x)$ must add to zero on each connected portion $\partial D_{n}$ of $\partial D$, again as exemplified by $(\mathrm{C} 7)$. For otherwise (C 9) would again be contradicted, the problem of inverting the Laplacian being equivalent to finding potential flow under the boundary condition (6.4) but this time with a non-vanishing circulation $\delta \gamma_{n}$ around at least one $\partial D_{n}$, implying a non-vanishing irrotational velocity field. (In terms of the membrane analogy one may imagine the membrane being attached to rigid wires having the shape of each $\partial D_{n}$, the wires being free to move normal to the equilibrium plane of the membrane in response to externally applied normal forces $\delta \gamma_{n}$ while remaining parallel to that plane. Again, the membrane evidently cannot remain flat.) It follows, then, that the $\delta q$ in question must be such that there is non-vanishing sheet or point vorticity distributed around at least one of the $\partial D_{n}$, but also such that this vorticity takes both positive and negative values, so as to add to zero. But we are given that the corresponding $\delta \mathscr{F} \neq 0$. The only way in which these statements can be consistent with (C 1) is for the value of $\delta \mathscr{F} / \delta q$ also to vary as we move around $\partial D_{n}$, i.e. for (C 5) to be violated on at least one of the $\partial D_{n}$. In summary, we have shown that $\mathscr{F}$ is admissible, i.e. satisfies (C 5), if and only if it is a functional of state in the sense defined above.

We note in addition that this statement still holds if $(6.6)$ is relaxed to constancy of circulation on $\partial D_{n}$, implying (6.5), since only changes $\delta u$ in the induced velocity field enter the argument just given, and $\delta \psi$ still satisfies (6.6).

Next, it is required to show that the Poisson bracket of any two admissible functionals $\mathscr{F}(q), \mathscr{G}(q)$ is itself admissible, that is to say

$$
\frac{\partial}{\partial s}\left(\frac{\delta}{\delta q}[\mathscr{F}, \mathscr{G}]\right)=0 \text { on } \partial D
$$

But this is an immediate corollary of what has just been said, since if $\mathscr{F}(q)$ and $\mathscr{G}(q)$

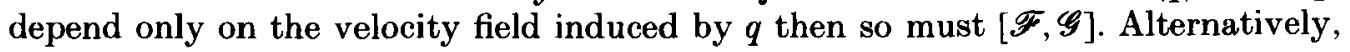


we may prove (C 10) directly as follows, at the same time preparing the way for the proof of Jacobi's identity. Writing $P(x)$ for $Q(x)+q(x)$, the basic-flow plus disturbance vorticity field, we first note from (7.24) that

$$
\begin{aligned}
\frac{\delta}{\delta q(\boldsymbol{x})}[\mathscr{F}, \mathscr{G}]=\partial\left(\frac{\delta \mathscr{F}}{\delta q(\boldsymbol{x})}, \frac{\delta \mathscr{G}}{\delta q(\boldsymbol{x})}\right)+ & \iint_{D} \frac{\delta^{2} \mathscr{F}}{\delta q(\boldsymbol{x}) \delta q(\hat{x})} \hat{\partial}\left(\frac{\delta \mathscr{G}}{\delta q(\hat{x})}, P(\hat{x})\right) \mathrm{d}^{2} \hat{x} \\
& -\iint_{D} \frac{\delta^{2} \mathscr{G}}{\delta q(\boldsymbol{x}) \delta q(\hat{x})} \hat{\partial}\left(\frac{\delta \mathscr{F}}{\delta q(\hat{x})}, P(\hat{x})\right) \mathrm{d}^{2} \hat{x}
\end{aligned}
$$

where $\hat{\partial}$ denotes the Jacobian with respect to the dummy variable $\hat{x}$. The first term on the right is the result of varying the function $q($.$) in the first factor of (7.24) only,$ using the rule

$$
\frac{\delta}{\delta q(\boldsymbol{x})} \iint P(\hat{x}) f(\hat{x}) \mathrm{d}^{2} \hat{x}=\frac{\delta}{\delta q(x)} \iint(Q(\hat{x})+q(\hat{x})) f(\hat{x}) \mathrm{d}^{2} \hat{x}=f(x)
$$

for an arbitrary function $f(x)$. This follows from (C 1). The second and third terms on the right of (C 11) come from using (7.25) to move $\delta \mathscr{F} / \delta q(\hat{x})$ and $\delta \mathscr{G} / \delta q(\hat{x})$ successively outside the Jacobian in (7.24), the dummy variable of integration in (7.24) being re-written as $\hat{x}$, and then applying $\delta / \delta q(x)$. If both $\mathscr{F}$ and $\mathscr{G}$ are admissible then the first term on the right of (C 11) vanishes when $x$ lies on $\partial D$, by (C 5) and the properties of Jacobians. In the second and third terms we have

$$
\frac{\delta^{2} \mathscr{F}}{\delta q(\boldsymbol{x}) \delta q(\hat{x})}=\frac{\delta}{\delta q(\hat{x})}\left(\frac{\delta \mathscr{F}\{q(.)\}}{\delta q(x)}\right)
$$

and similarly for $\mathscr{G}$, by (C 4). But if $x$ is now restricted to lie on one of the $\partial D_{n}$, then (C 5), or (C 6), implies that $\delta \mathscr{F}\{q().\} / \delta q(x)$ depends only on the function $q($.$) and not$ on the position of the point $\boldsymbol{x}$ on $\partial D_{n}$. It follows that the right-hand side of (C 13) is then a function of $\hat{\boldsymbol{x}}$ alone, and not of $\boldsymbol{x}$, and hence that the second term on the right-hand side of $(\mathrm{C} 11)$ is constant as required, when $x$ varies over $\partial D_{n}$. The third term is similarly constant, and so the whole expression (C 11) is constant on $\partial D_{n}$. Therefore $(\mathrm{C} 10)$ is true.

To prove Jacobi's identity $(7.26 c)$ for three admissible functionals $\mathscr{F}, \mathscr{G}$ and $\mathscr{K}$ we need to substitute (C 11) into the expression

$$
\iint P(x) \partial\left(\frac{\delta[\mathscr{F}, \mathscr{G}]}{\delta q(x)}, \frac{\delta \mathscr{K}}{\delta q(x)}\right) \mathrm{d}^{2} x
$$

add the result to its two cyclic permutations, and show that the sum of all three expressions is zero. Now the contribution from the first term on the right-hand side of (C 11) vanishes separately, because the Jacobian itself satisfies its own Jacobi identity

$$
\partial((f, g), h)+\partial((g, h), f)+\partial((h, f), g)=0,
$$

for any three functions $f(x), g(x)$ and $h(x)$, as is easily verified. For the remaining contribution it is easier to rewrite (C 14) as

$$
\iint \frac{\delta[\mathscr{F}, \mathscr{G}]}{\delta q(\boldsymbol{x})} \partial\left(\frac{\delta \mathscr{K}}{\delta q(\boldsymbol{x})}, P(\boldsymbol{x})\right) \mathrm{d}^{2} \boldsymbol{x}
$$


again using (7.25) and the admissibility of [F, $\mathscr{G}]$. Substitution from the last two terms of (C 11) shows that the remaining contribution can be written

$$
\begin{aligned}
& \iiint \int_{D \times D} \mathrm{~d}^{2} x \mathrm{~d}^{2} \hat{x} \frac{\delta^{2} \mathscr{F}}{\delta q(\boldsymbol{x}) \delta q(\hat{x})} \hat{\partial}\left(\frac{\delta \mathscr{G}}{\delta q(\hat{x})}, P(\hat{x})\right) \partial\left(\frac{\delta \mathscr{K}}{\delta q(\boldsymbol{x})}, P(x)\right) \\
& \quad-\iiint \int_{D \times D} \mathrm{~d}^{2} x \mathrm{~d}^{2} \hat{x} \frac{\delta^{2} \mathscr{G}}{\delta q(\boldsymbol{x}) \delta q(\hat{x})} \hat{\partial}\left(\frac{\delta \mathscr{F}}{\delta q(\hat{x})}, P(\hat{x})\right) \partial\left(\frac{\delta \mathscr{K}}{\delta q(x)}, P(x)\right)+\ldots,
\end{aligned}
$$

where the dots denote two further pairs of similar terms produced by cyclicly permuting $\mathscr{F}, \mathscr{G}$ and $\mathscr{K}$. It can now be seen from (C 4) that the six terms cancel in pairs.

It remains to show that both (C 10), and Jacobi's identity $(\mathbf{7 . 2 6} c)$, still hold if the space of admissible functionals is extended to include Casimirs. Because of bilinearity, and the fact that all Casimirs were shown to have the form (7.12), it is sufficient to verify (C 10) and (7.26c) when one, two, or all three of $\mathscr{F}, \mathscr{G}$ and $\mathscr{K}$ are such that

$$
\frac{\delta \mathscr{F}}{\delta q(x)}=\text { func }\{P(x)\}
$$

etc., 'func' denoting an ordinary function, and the others, if any, satisfy the admissibility condition (C 5). First, it is straightforward to verify that in all three cases, (7.25) still holds when, say, $f(x)=P(x)$ and $g, h$ are selected from $\delta \mathscr{F} / \delta q, \delta \mathscr{G} / \delta q$ and $\delta \mathscr{K} / \delta q$. Thus cyclic permutation in (7.24) is still permissible, and it follows immediately that $[\mathscr{F}, \mathscr{G}]$ vanishes if either or both of $\mathscr{F}, \mathscr{G}$ satisfy (C 18). This can also be seen directly by changing variables in (7.24). It follows similarly that each term in $(7.26 c)$ vanishes, in all three cases of interest. Thus both $(C 10)$ and $(7.26 c)$ are satisfied trivially.

A still wider extension of the class of admissible functionals is considered in the recent paper of Lewis et al. (1986). Their extension is important in problems where the boundary $\partial D$ is not rigid, e.g. a free surface, in which case certain boundaryintegral terms must be added to (7.24).

\section{REFERENCES}

Abarbanel, H. D. I., Holm, D. D., Marsden, J. E. \& Ratiu, T. 1984 Richardson number criterion for the nonlinear stability of three-dimensional flow. Phys. Rev. Lett. 52, 2352-2355.

Abarbanel, H. D. I., Holm, D. D., Marsden, J. E. \& Ratiu, T. 1986 Nonlinear stability analygis of stratified fluid equilibria. Phil. Trans. R. Soc. Lond. A 318, 349-409.

Abraham, R. \& Marsden, J. E. 1978 Foundations of Mechanics, 2nd edn. Benjamin/Cummings, $806 \mathrm{pp}$.

Al-AJMI, D. N., Harwood, R. S. \& Miles, T. 1985 A sudden warming in the middle atmosphere of the southern hemisphere. Q. J. R. Met. Soc. 111, 359-389.

ANDREws, D. G. 1981 A note on potential energy density in a stratified compressible fluid. J. Fluid Mech. 107, 227-236.

ANDREws, D. G. 1983 A conservation law for small-amplitude quasi-geostrophic disturbances on a zonally asymmetric basic flow. J. Atmos. Sci. 40, 85-90.

ANDRews, D. G. \& McIstyre, M. E. 1978 a An exact theory of nonlinear waves on a Lagrangianmean flow. J. Fluid Mech. 89, 609-646.

Andrews, D. G. \& McIntyre, M. E. $1978 b$ On wave-action and its relatives. J. Fluid Mech. 89, 647-664 (and Corrigenda 95, 796).

ARnol'D, V. I. 1965 Conditions for nonlinear stability of stationary plane curvilinear flows of an ideal fluid. Dokl. Akad. Nauk. SSSR 162, 975-978. (English transl.: Soviet Math. 6, 773-777 (1965).) 
ARnoL'D, V. I. 1966a On an a priori estimate in the theory of hydrodynamical stability. Izv. Vyssh. Uchebn. Zaved. Matematika 54, no. 5, 3-5. (English transl.: American Math. Soc. Transl., Series $279,267-269$ (1969).)

ARnol'D, V. I. $1966 b$ Sur la géométrie différentielle des groupes de Lie de dimension infinie et ses applications à l'hydrodynamique des fluides parfaits. Ann. Inst. Fourier (Grenoble) 16, 319-361.

ARnol'D, V. I. 1969 The Hamiltonian nature of the Euler equations in the dynamics of a rigid body and of a perfect fluid. Usp. Mat. Nauk. 24, no. 3, 225-226. (In Russian.) (Rough translation available from authors.)

ARnol'D, V. I. 1978 Mathematical Methods of Classical Mechanics. Springer, 462 pp. (Russian original: Nauka, 1974.)

BALL, J. M. \& MARSDen, J. E. 1984 Quasiconvexity at the boundary, positivity of the second variation, and elastic stability. Arch. Rat. Mech. Anal. 86, 251-277.

Batchelor, G. K. 1967 An Introduction to Fluid Dynamics. Cambridge University Press, 615 pp.

Benjamin, T. B. 1972 The stability of solitary waves. Proc. R. Soc. Lond. A 328, 153-183.

BENJAMIN, T. B. 1984 Impulse, flow force and variational principles. IMA J. Appl. Maths 32, 3-68.

Benjamin, T. B. 1986 On the Boussinesq model for two-dimensional wave motions in heterogeneous fluids. J. Fluid Mech. 165, 445-474.

Blumen, W. 1968 On the stability of quasi-geostrophic flow. J. Atmos. Sci. 25, 929-931.

Boyd, J. P. 1983 The continuous spectrum of linear Couette flow with the beta effect. J. Atmos. Sci. 40, 2304-2308.

Bretherton, F. P. 1966 a Critical layer instability in baroclinic flows. Q. J. R. Met. Soc. 92, 325-334.

Bretherton, F. P. $1966 b$ Baroclinic instability and the short wavelength cut-off in terms of potential vorticity. Q. J. R. Met. Soc. 92, 335-345.

Bretherton, F. P. 1970 A note on Hamilton's principle for perfect fluids. J. Fluid Mech. 44, 19-31.

Bretherton, F. P. \& Garretr, C. J. R. 1968 Wavetrains in inhomogeneous moving media. Proc. R. Soc. Lond. A 302, 529-554.

Butchart, N. \& Remsber, E. E. 1986 The area of the stratospheric polar vortex as a diagnostic for tracer transport on an isentropic surface. J. Atmos. Sci. 43, 1319-1339.

Charney, J. G. 1973 Planetary fluid dynamics. In Dynamic Meteorology (ed. P. Morel), pp. 97-351. Reidel.

Charney, J. G. \& DeVore, J. G. 1979 Multiple flow equilibria in the atmosphere and blocking. J. Atmos. Sci. 36, 1205-1216.

Charney, J. G. \& Flierl, G. R. 1981 Oceanic analogues of large-scale atmospheric motions. In Evolution of Physical Oceanography (ed. B. A. Warren \& C. Wunsch), pp. 504-548. M.I.T. Press.

Charney, J. G. \& Pedlosky, J. 1963 On the trapping of unstable planetary waves in the atmosphere. J. Geophys. Res. 68, 6441-6442.

Clovgh, S. A., Grahame, N. S. \& O'NeIll, A. 1985 Potential vorticity in the stratosphere derived using data from satellites. Q. J. R. Met. Soc. 111, 335-358.

DeEm, G. S. \& ZABUSKY, N. J. 1978 Vortex waves: stationary ' $V$ states', interactions, recurrence, and breaking. Phys. Rev. Lett. 40, 859-862.

Dikiy, L. A. \& KuraAnskiy, M. V. 1971 Integral conservation law for perturbations of zonal flow, and its application to stability studies. Izv. FAO 7, 939-945. (English transl.: Atmos. Ocean. Phys. 7, 623-626 (1971).)

Drrac, P. A. M. 1958 The Principles of Quantum Mechanics, 4th edn. Oxford University Press, $312 \mathrm{pp}$.

DrITSCHEL, D. G. 1986 The nonlinear evolution of rotating configurations of uniform vorticity. J. Fluid Mech. 172, 157-182.

Dunkerton, T. J. \& Delisi, D. P. 1986 Evolution of potential vorticity in the winter stratosphere of January-February 1979. J. Geophys. Res. 91 (D1), 1199-1208.

FarReLL, B. F. 1982 The initial growth of disturbances in a baroclinic flow. J. Atmos. Sci. 39, 1663-1686. 
FJøRroft, R. 1953 On the changes in the spectral distribution of kinetic energy for two dimensional, nondivergent flow. Tellus 5, 225-230.

Goldstein, H. 1980 Classical Mechanics, 2nd edn. Addison-Wesley, 672 pp.

Grose, W. L. 1984 Recent advances in understanding stratospheric dynamics and transport processes: Application of satellite data to their interpretation. Adv. Space Res. 4, 19-28.

Haynes, P. H. 1985 Nonlinear instability of a Rossby-wave critical layer. J. Fluid Mech. 161, $493-511$.

HAYNES, P. H. 1987 On the instability of sheared disturbances. J. Fluid Mech. 175, 463-478.

Hold, I. M. 1985 Pseudomomentum and the orthogonality of modes in shear flows. J. Atmos Sci. 42, 2280-2288.

Hirota, I. 1967 Dynamic stability of the stratospheric polar vortex. J. Met. Soc. Japan 45, 409-421.

Hrrsch, M. W. \& Smale, S. 1974 Differential Equations, Dynamical Systems, and Linear Algebra. London: Academic Press, $358 \mathrm{pp}$.

Holliday, D. \& McIntyke, M. E. 1981 On potential energy density in an incompressible, stratified fluid. J. Fluid Mech. 107, 221-225.

Holm, D. D. 1986 Hamiltonian formulation of the baroclinic quasigeostrophic fluid equations. Phys. Fluids 29, 7-8.

Holm, D. D., Marsden, J. E., Rativ, T. \& Weinstein, A. 1983 Nonlinear stability conditions and a priori estimates for barotropic hydrodynamics. Phys. Lett. 98A, 15-21.

Holm, D. D., Marsden, J. E., Rativ, T. \& Weinstein, A. 1985 Nonlinear stability of fluid and plasma equilibria. Phys. Rep. 123, 1-116.

Hoskins, B. J., McIntyre, M. E. \& Robertson, A. W. 1985 On the use and significance of isentropic potential-vorticity maps. Q.J. R. Met. Soc. 111, 877-946. Also 113, 402-404.

Juckes, M. N. \& McIntyre, M. E. 1987 A high resolution, one-layer model of breaking planetary waves in the stratosphere. Nature (to appear).

Killworth, P. D. \& McINTYRe, M. E. 1985 Do Rossby-wave critical layers absorb, reflect or over-reflect? J. Fluid Mech. 161, 449-492.

Kuznetsov, E. A. \& Mikhailov, A. V. 1980 On the topological meaning of canonical Clebsch variables. Phys. Lett. 77A, 37-38.

Lamb, H. 1932 Hydrodynamics, 6th edn. Cambridge University Press, 738 pp.

Leovy, C. B., Sun, C.-R., Hitchman, M. H., Remsberg, E. E., Russell, J. M., Gordley, L. L., GiLle, J. C. \& LyJaK, L. V. 1985 Transport of ozone in the middle stratosphere: evidence for planetary wave breaking. J. Atmos. Sci. 42, 230-244.

Lewis, D., Marsden, J. E., Montgomery, R. \& Ratiu, T. 1986 The Hamiltonian structure for dynamic free boundary problems. Physica 18D, 391-404.

LightнiLl, M. J. 1963 Introduction. Boundary layer theory. In Laminar Boundary Layers (ed. L. Rosenhead), pp. 46-113. Oxford University Press.

Lonavet-Higgins, M. S. 1964 On group velocity and energy flux in planetary wave motions. Deep-Sea Res. 11, 35-42.

McIntyre, M. E. 1987 The Lagrangian description in fluid mechanics. IMA J. Appl. Math., to be published.

McIntyre, M. E. \& Palmer, T. N. 1983 Breaking planetary waves in the stratosphere. Nature $305,593-600$.

McIntyre, M. E. \& Palmer, T. N. 1984 The 'surf zone' in the stratosphere. J. Atmos. Terres. Phys. 46, 825-849.

McIntyre, M. E. \& Palmer, T. N. 1985 A note on the general concept of wave breaking for Rossby and gravity waves. Pure Appl. Geophys. 123, 964-975.

McINTYRe, M. E. \& Weissman, M. A. 1978 On radiating instabilities and resonant overreflection. J. Atmos. Sci. 35, 1190-1198.

Marsden, J. E. (ed.) 1984 Fluids and Plasmas: Geometry and Dynamics. Contemporary Mathematics, vol. 28, pp. vii-xiii. American Mathematical Society.

MARSDEN, J. E. \& Weinstein, A. 1983 Coadjoint orbits, vortices, and Clebsch variables for incompressible fuids. Physica 7D, 305-323. 
Matsuno, T. \& Hirota, 1. 1966 On the dynamical stability of polar vortex in wintertime. $J$. Met. Soc. Japan 44, 122-128.

Merilees, P. E. \& WARn, H. 1975 On energy and enstrophy exchanges in two-dimensional non-divergent flow. J. Fluid Mech. 69, 625-630.

Morrison, P. J. 1982 Poisson brackets for fluids and plasmas. In Mathematical Methods in Hydrodynamics and Integrability in Dynamical Systems (ed. M. Tabor \& Y. M. Treve), Amer. Inst. Phys. Conf. Proc. vol. 88, pp. 13-46.

Olver, P. J. 1980 On the Hamiltonian structure of evolution equations. Math. Proc. Camb. Phil. Soc. 88, 71-88.

Olver, P. J. 1982 A nonlinear Hamiltonian structure for the Euler equations. J. Math. Anal. Appl. $89,233-250$.

OLVER, P. J. 1983 Conservation laws of free boundary problems and the classification of conservation laws for water waves. Trans. Am. Math. Soc. 277, 353-380.

OLVer, P. J. 1984 Hamiltonian perturbation theory and water waves. In Fluids and Plasmas: Geometry and Dynamics (ed. J. E. Marsden). Contemporary Mathematics, vol. 28, pp. 231-249. American Mathematical Society.

ORR, W. M'F. 1907 The stability or instability of the steady motions of a perfect liquid and of a viscous liquid. Parts I and II. Proc. R. Irish Acad. A27, 9-68; 69-138.

Pedlosky, J. 1979 Geophysical Fluid Dynamics. Springer, 624 pp.

Penfield, P. 1966 Hamilton's principle for fluids. Phys. Fluids 9, 1184-1194.

Plumb, R. A. 1979 Forced waves in a baroclinic shear flow. Part 1: Undamped evolution near the baroclinic instability threshold. $J$. Atmos. Sci. 36, 205-216.

Plumb, R. A. 1981 Instability of the distorted polar night vortex: A theory of stratospheric warmings. J. Atmos. Sci. 38, 2514-2531.

Pudmi, R. A. $1985 a$ On the three-dimensional propagation of stationary waves. J. Atmos. Sci. 42, 217-229.

Plumb, R. A. $1985 b$ An alternative form of Andrews' conservation law for quasi-geostrophic waves on a steady, nonuniform flow. J. Atmos. Sci. 42, 298-300.

RIPA, P. 1981 Symmetries and conservation laws for internal gravity waves. In Nonlinear Properties of Internal Waves (ed. B. J. West), Amer. Inst. Phys. Conf. Proc. vol. 76, pp. 281-306.

Salmon, R. 1982 Hamilton's principle and Ertel's theorem. In Mathematical Methods in Hydrodynamics and Integrability in Dynamical Systems (ed. M. Tabor \& Y. M. Treve), Amer. Inst. Phys. Conf. Proc. vol. 88, pp. 127-135.

Salmon, R. 1988 Hamiltonian fluid mechanics. Ann. Rev. Fluid Mech. (to appear).

SchIFF, L. I. 1968 Quantum Mechanics, 3rd edn. McGraw-Hill, 544 pp.

Shepherd, T. G. 1985 Time development of small disturbances to plane Couette flow. J. Atmos. Sci. 42, 1868-1871.

Stewartson, K. 1978 The evolution of the critical layer of a Rossby wave. Geophys. Astrophys. Fluid Dyn. 9, 185-200.

Stuart, J. T. 1967 On finite amplitude oscillations in laminar mixing layers. J. Fluid Mech. 29, 417-440.

Sudarshan, E. C. G. \& Mukunda, N. 1974 Classical Dynamics: A Modern Perspective. WileyInterscience, $615 \mathrm{pp}$.

Swaters, G. E. 1986 A nonlinear stability theorem for baroclinic quasigeostrophic flow. Phys. Fluids 29, 5-6.

Tномson, W. 1887 Stability of fluid motion - rectilineal motion of viscous fluid between two parallel planes. Phil. Mag. 24, 188-196.

Wan, Y. H. \& Pulvirenti, M. 1985 Nonlinear stability of circular vortex patches. Commun. Math. Phys. 99, 435-450.

W ARn, T. \& WARN, H. 1978 The evolution of a nonlinear critical level. Stud. Appl. Math. 59, $37-71$.

Whittaker, E. T. 1937 A Treatise on the Analytical Dynamics of Particles and Rigid Bodies, 4th edn. Cambridge University Press, 456 pp. 